\title{
VAR for VaR: Measuring Tail Dependence Using Multivariate Regression Quantiles*
}

\author{
Halbert White Tae-Hwan Kim $^{\ddagger} \quad$ Simone Manganelli ${ }^{\S}$
}

August 8, 2012

\begin{abstract}
This paper proposes methods for estimation and inference in multivariate, multi-quantile models. The theory can simultaneously accommodate models with multiple random variables, multiple confidence levels, and multiple lags of the associated quantiles. The proposed framework can be conveniently thought of as a vector autoregressive (VAR) extension to quantile models. We estimate a simple version of the model using market equity returns data to analyse spillovers in the values at risk (VaR) between a market index and financial institutions. We construct impulse-response functions for the quantiles of a sample of 230 financial institutions around the world and study how financial institution-specific and system-wide shocks are absorbed by the system. We show how our methodology can successfully identify both in-sample and out-of-sample the set of financial institutions whose risk is most sentitive to market wide shocks in situations of financial distress, and can prove a valuable addition to the traditional toolkit of policy makers and supervisors.

Keywords: Quantile impulse-responses, spillover, codependence, CAViaR

JEL classification: C13, C14, C32.

\footnotetext{
${ }^{*}$ We would like to thank Peter Christoffersen, Rob Engle, Rossen Valkanov, as well seminar participants at the ECB, the Fourth Annual SoFiE Conference in Chicago, the Cass Business School, the London School of Business, the Fourth Tremblant Risk Management Conference, the Vienna Graduate School of Finance and the Fourteenth International Korean Economic Association Conference. Francesca Fabbri and Thomas Kostka provided data support. The phrase "VAR for VaR" was first used by Andersen et al. (2003), in the title of their section 6.4. The views expressed in this paper are those of the authors and do not necessarily reflect those of the European Central Bank or the Eurosystem. Tae-Hwan Kim is grateful for the financial support from the National Research Foundation of Korea - a grant funded by the Korean Government (NRF-2009-327-B00088).

${ }^{\dagger}$ Department of Economics, University of California, San Diego.

${ }^{\ddagger}$ School of Economics, Yonsei University.

${ }^{\S}$ Corresponding author. European Central Bank, DG-Research, Kaiserstrasse 29, 60433 Frankfurt am Main, Germany. Email: simone.manganelli@ecb.int.
} 


\section{Introduction}

This paper suggests a multivariate regression quantile model to directly study the degree of tail interdependence among different random variables. Our theoretical framework allows the quantiles of several random variables to depend on (lagged) quantiles, as well as past innovations and other covariates of interest. This modeling strategy has at least three advantages over the more traditional approaches that rely on the parameterization of the entire multivariate distribution. First, regression quantile estimates are known to be robust to outliers, a desirable feature in general and for applications to financial data in particular. Second, regression quantile is a semi-parametric technique and as such imposes minimal distributional assumptions on the underlying data generating process (DGP). Third, our multivariate framework allows researchers to directly measure the tail dependence among the random variables of interest, rather than recovering it indirectly via models of time-varying first and second moments.

To illustrate our approach and its usefulness, consider a simple set-up with two random variables, $Y_{1 t}$ and $Y_{2 t}$. All information available at time $t$ is represented by the information set $\mathcal{F}_{t-1}$. For a given level of confidence $\theta \in$ $(0,1)$, the quantile $q_{i t}$ at time $t$ for random variables $Y_{i t} i=1,2$ conditional on $\mathcal{F}_{t-1}$ is

$$
\operatorname{Pr}\left[Y_{i t} \leq q_{i t} \mid \mathcal{F}_{t-1}\right]=\theta, \quad i=1,2 .
$$

A simple version of our proposed structure relates the conditional quantiles of the two random variables according to a vector autoregressive (VAR) structure:

$$
\begin{aligned}
& q_{1 t}=X_{t}^{\prime} \beta_{1}+b_{11} q_{1 t-1}+b_{12} q_{2 t-1}, \\
& q_{2 t}=X_{t}^{\prime} \beta_{2}+b_{21} q_{1 t-1}+b_{22} q_{2 t-1},
\end{aligned}
$$

where $X_{t}$ represents predictors belonging to $\mathcal{F}_{t-1}$ and typically includes lagged values of $Y_{i t}$. If $b_{12}=b_{21}=0$, the above model reduces to the univariate CAViaR model of Engle and Manganelli (2004), and the two specifications can be estimated independently from each other. If, however, $b_{12}$ and/or $b_{21}$ are different from zero, the model requires the joint estimation of all of the parameters in the system. The off-diagonal coefficients $b_{12}$ and $b_{21}$ represent the measure of tail codependence between the two random variables, thus the hypothesis of no tail codependence can be assessed by testing $H_{0}: b_{12}=b_{21}=0$.

The first part of this paper develops the consistency and asymptotic theory for the multivariate regression quantile model. Our fully general model is much richer than the above example, as we can accommodate: (i) more than two random variables; (ii) multiple lags of $q_{i t}$; and (iii) multiple confidence levels, say $\left(\theta_{1}, \ldots, \theta_{p}\right)$. 
In the second part of this paper, as an empirical illustration of the model, we estimate a series of bivariate VAR models for the conditional quantiles of the return distributions of individual financial institutions from around the world. Since quantiles represent one of the key inputs for the computation of the Value at Risk (VaR) for financial assets, we call this model VAR for $\mathrm{VaR}$, that is, a vector autoregressive (VAR) model where the dependent variables are the $\mathrm{VaR}$ of the financial institutions, which are dependent on (lagged) VaR and past shocks.

Our modeling framework appears particularly suitable to develop sound measures of financial spillover, the importance of which has been brought to the forefront by the recent financial crisis. In the current policy debate, great emphasis has been put on how to measure the additional capital needed by financial institutions in a situation of generalized market distress. The logic is that if the negative externality associated with the spillover of risks within the system is not properly internalised, banks may find themselves in need of additional capital at exactly the worst time, such as when it is most difficult and expensive to raise fresh new capital. If the stability of the whole system is threatened, taxpayer money has to be used to backstop the financial system, to avoid systemic bank failures that may bring the whole economic system to a collapse.

Adrian and Brunnermeier (2009), Acharya et al. (2009), and Brownlees and Engle (2010) have recently proposed to classify financial institutions according to the sensitivity of their VaR to shocks to the whole financial system. The empirical section of this paper illustrates how the multivariate regression quantile model provides an ideal framework to estimate directly the sensitivity of VaR of a given financial institution to system-wide shocks. A useful by-product of our modeling strategy is the ability to compute quantile impulse-response functions. These are obtained by computing the long run quantiles and then applying a suitable identified shock to the multivariate quantile model. Using the quantile impulse-response functions, we can assess the resilience of financial institutions to shocks to the overall index, as well as their persistence.

The model is estimated on a sample of 230 financial institutions from around the world. For each of these equity return series, we estimate a bivariate VAR for VaR where one variable is the return on a portfolio of financial institutions and the other variable is the return on the single financial institution. We find strong evidence of significant tail codependence for a large fraction of the financial institutions in our sample. When aggregating the impulse response functions at the sectoral and geographic level no striking differences are revealed. We, however, find significant cross-sectional differences. By aggregating the 20 stocks with the strongest and weakest tail codependence to market shocks (thus, forming two portfolios), we find that, while in tranquil times, the two portfolios have comparable risk. In times of severe financial distress, the risk of the first portfolio increases dis- 
proportionately relative to the second. This result holds for both in-sample and out-of-sample.

The plan of this paper is as follows. In Section 2, we set forth the multivariate and multi-quantile CAViaR framework, a generalization of Engle and Manganelli's original CAViaR (2004) model. Section 3 provides conditions ensuring the consistency and asymptotic normality of the estimator, as well as the results which provide a consistent asymptotic covariance matrix estimator. Section 4 contains our empirical study. Section 5 provides a summary and some concluding remarks. The appendix contains all of the technical proofs of the theorems in the text.

\section{The Multivariate and Multi-Quantile Process and Its Model}

We consider a sequence of random variables denoted by $\left\{\left(Y_{t}^{\prime}, X_{t}^{\prime}\right): t=\right.$ $1,2, \ldots, T\}$ where $Y_{t}$ is a finitely dimensioned $n \times 1$ vector and $X_{t}$ is also a countably dimensioned vector whose first element is one. To fix ideas, $Y_{t}$ can be considered as the dependent variables and $X_{t}$ as the explanatory variables in a typical regression framework. In this sense, the proposed model which will be developed below is sufficiently general enough to handle multiple dependent variables. We specify the data generating process as follows.

Assumption 1 The sequence $\left\{\left(Y_{t}^{\prime}, X_{t}^{\prime}\right)\right\}$ is a stationary and ergodic stochastic process on the complete probability space $\left(\Omega, \mathcal{F}, P_{0}\right)$, where $\Omega$ is the sample space, $\mathcal{F}$ is a suitably chosen $\sigma$-field, and $P_{0}$ is the probability measure providing a complete description of the stochastic behavior of the sequence of $\left\{\left(Y_{t}^{\prime}, X_{t}^{\prime}\right)\right\}$.

We define $\mathcal{F}_{t-1}$ to be the $\sigma$-algebra generated by $Z^{t-1}:=\left\{X_{t},\left(Y_{t-1}, X_{t-1}\right), \ldots\right\}$, i.e. $\mathcal{F}_{t-1}:=\sigma\left(Z^{t-1}\right)$. For $i=1, \ldots, n$, we also define $F_{i t}(y):=P_{0}\left[Y_{i t}<y \mid\right.$ $\left.\mathcal{F}_{t-1}\right]$ which is the cumulative distribution function $(\mathrm{CDF})$ of $Y_{i t}$ conditional on $\mathcal{F}_{t-1}$. In the quantile regression literature, it is typical to focus on a specific quantile index; for example, $\theta \in(0,1)$. In this paper, we will develop a more general quantile model where multiple quantile indexes can be accounted for jointly. To be more specific, we consider $p$ quantile indexes denoted by $\theta_{i 1}, \theta_{i 2}, \ldots, \theta_{i p}$ for the $i t h$ element (denoted by $Y_{i t}$ ) of $Y_{t}$. The $p$ quantile indexes do not need to be the same for all of the elements of $Y_{t}$, which explains the double indexing of $\theta_{i j}$. Moreover, we note that we specify the same number $(p)$ of quantile indexes for each $i=1, \ldots, n$. However, this is just for notational simplicity. Our theory easily applies to the case in which the number of quantile indexes differs with $i$; i.e., $p$ can be replaced with $p_{i}$.

To formalize our argument, we assume that the quantile indexes are 
ordered such that $0<\theta_{i 1}<\ldots<\theta_{i p}<1$. For $j=1, \ldots, p$, the $\theta_{i j}$ th quantile of $Y_{i t}$ conditional on $\mathcal{F}_{t-1}$, denoted $q_{i, j, t}^{*}$, is

$$
q_{i, j, t}^{*}:=\inf \left\{y: F_{i t}(y)=\theta_{i j}\right\}
$$

and if $F_{i t}$ is strictly increasing,

$$
q_{i, j, t}^{*}=F_{i t}^{-1}\left(\theta_{i j}\right) .
$$

Alternatively, $q_{i, j, t}^{*}$ can be represented as

$$
\int_{-\infty}^{q_{i, j, t}^{*}} d F_{i t}(y)=E\left[1_{\left[Y_{i t} \leq q_{i, j, t}^{*}\right]} \mid \mathcal{F}_{t-1}\right]=\theta_{i j}
$$

where $d F_{i t}(\cdot)$ is the Lebesgue-Stieltjes probability density function (PDF) of $Y_{i t}$ conditional on $\mathcal{F}_{t-1}$, corresponding to $F_{i t}$.

Our objective is to jointly estimate the conditional quantile functions $q_{i, j, t}^{*}$ for $i=1, \ldots, n$ and $j=1,2, \ldots, p$. For this, we write $q_{t}^{*}:=\left(q_{1, t}^{* \prime}, q_{2, t}^{* \prime}, \ldots, q_{n, t}^{* \prime}\right)^{\prime}$ with $q_{i, t}^{*}:=\left(q_{i, 1, t}^{*}, q_{i, 2, t}^{*}, \ldots, q_{i, p, t}^{*}\right)^{\prime}$ and impose an additional appropriate structure. First, we ensure that the conditional distributions of $Y_{i t}$ are everywhere continuous, with positive densities at each of the conditional quantiles of interest, $q_{i, j, t}^{*}$. We let $f_{i t}$ denote the conditional probability density function (PDF) which corresponds to $F_{i t}$. In stating our next condition (and where helpful elsewhere), we make explicit the dependence of the conditional CDF $F_{i t}$ on $\omega \in \Omega$ by writing $F_{i t}(\omega, y)$ in place of $F_{i t}(y)$. Similarly, we may write $f_{i, t}(\omega, y)$ in place of $f_{i, t}(y)$. The realized values of the conditional quantiles are correspondingly denoted $q_{i, j, t}^{*}(\omega)$.

Our next assumption ensures the desired continuity and imposes specific structure on the quantiles of interest.

Assumption 2 (i) $Y_{i t}$ is continuously distributed such that for each $\omega \in$ $\Omega, F_{i t}(\omega, \cdot)$ and $f_{i t}(\omega, \cdot)$ are continuous on $\mathbb{R}, t=1,2, \ldots, T$; (ii) For the given $0<\theta_{i 1}<\ldots<\theta_{i p}<1$ and $\left\{q_{i, j, t}^{*}\right\}$ as defined above, we suppose the following: (a) for each $i, j, t$, and $\omega, f_{i t}\left(\omega, q_{i, j, t}^{*}(\omega)\right)>0$; and (b) for the given finite integers $k$ and $m$, there exist a stationary ergodic sequence of random $k \times 1$ vectors $\left\{\Psi_{t}\right\}$, with $\Psi_{t}$ measurable $-\mathcal{F}_{t-1}$, and real vectors $\beta_{i j}^{*}:=\left(\beta_{i, j, 1}^{*}, \ldots, \beta_{i, j, k}^{*}\right)^{\prime}$ and $\gamma_{i, j, \tau}^{*}:=\left(\gamma_{i, j, \tau, 1}^{* \prime}, \ldots, \gamma_{i, j, \tau, n}^{* \prime}\right)^{\prime}$, where each $\gamma_{i, j, \tau, k}^{*}$ is a $p \times 1$ vector, such that for $i=1, \ldots, n, j=1, \ldots, p$, and all $t$,

$$
q_{i, j, t}^{*}=\Psi_{t}^{\prime} \beta_{i j}^{*}+\sum_{\tau=1}^{m} q_{t-\tau}^{* \prime} \gamma_{i, j, \tau}^{*} .
$$

The structure of equation in (4) is a multivariate version of the MQCAViaR process of White, Kim, and Manganelli (2008), itself a multiquantile version of the CAViaR process introduced by Engle and Manganelli 
(2004). Under suitable restrictions on $\gamma_{i, j, \tau}^{*}$, we obtain as special cases; (1) separate MQ-CAViaR processes for each element of $Y_{t}$; (2) standard (single quantile) CAViaR processes for each element of $Y_{t}$; or (3) multivariate CAViaR processes, in which a single quantile of each element of $Y_{t}$ is related dynamically to the single quantiles of the (lags of) other elements of $Y_{t}$. Thus, we call any process that satisfies our structure "Multivariate MQ-CAViaR" (MVMQ-CAViaR) processes or naively "VAR for VaR."

For MVMQ-CAViaR, the number of relevant lags can differ across the elements of $Y_{t}$ and the conditional quantiles; this is reflected in the possibility that for the given $j$, elements of $\gamma_{i, j, \tau}^{*}$ may be zero for values of $\tau$ greater than some given integer. For notational simplicity, we do not represent $m$ as being dependent on $i$ or $j$. Nevertheless, by convention, for no $\tau \leq m$ does $\gamma_{i, j, \tau}^{*}$ equal the zero vector for all $i$ and $j$. The finitely dimensioned random vectors $\Psi_{t}$ may contain lagged values of $Y_{t}$, as well as measurable functions of $X_{t}$ and lagged $X_{t}$. In particular, $\Psi_{t}$ may contain Stinchcombe and White's (1998) GCR transformations, as discussed in White (2006).

For a particular quantile, say $\theta_{i j}$, the coefficients to be estimated are $\beta_{i j}^{*}$ and $\gamma_{i j}^{*}:=\left(\gamma_{i, j, 1}^{* \prime}, \ldots, \gamma_{i, j, m}^{* \prime}\right)^{\prime}$. Let $\alpha_{i j}^{* \prime}:=\left(\beta_{i j}^{* \prime}, \gamma_{i j}^{* \prime}\right)$, and write $\alpha^{*}=$ $\left(\alpha_{11}^{* \prime}, \ldots, \alpha_{1 p}^{* \prime}, \ldots, \alpha_{n 1}^{* \prime}, \ldots\right.$,

$\left.\alpha_{n p}^{* \prime}\right)^{\prime}$, an $\ell \times 1$ vector, where $\ell:=n p(k+n p m)$. We call $\alpha^{*}$ the "MVMQCAViaR coefficient vector." We estimate $\alpha^{*}$ using a correctly specified model for the MVMQ-CAViaR process. First, we specify our model.

Assumption 3 (i) Let $\mathbb{A}$ be a compact subset of $\mathbb{R}^{\ell}$. For $i=1, \ldots, n$, and $j=1, \ldots, p$, we suppose the following: (a) the sequence of functions $\left\{q_{i, j, t}: \Omega \times \mathbb{A} \rightarrow \mathbb{R}^{p_{i}}\right\}$ is such that for each $t$ and each $\alpha \in \mathbb{A}, q_{i, j, t}(\cdot, \alpha)$ is measurable $-\mathcal{F}_{t-1}$; (b) for each $t$ and each $\omega \in \Omega, q_{i, j, t}(\omega, \cdot)$ is continuous on $\mathbb{A}$; and (c) for each $i, j$, and $t, q_{i, j, t}(\cdot, \alpha)$ is specified as follows:

$$
q_{i, j, t}(\cdot, \alpha)=\Psi_{t}^{\prime} \beta_{i j}+\sum_{\tau=1}^{m} q_{t-\tau}(\cdot, \alpha)^{\prime} \gamma_{i, j, \tau} .
$$

Next, we impose the correct specification assumption together with an identification condition. Assumption 4(i.a) below delivers the correct specification by ensuring that the MVMQ-CAViaR coefficient vector $\alpha^{*}$ belongs to the parameter space, $\mathbb{A}$. This ensures that $\alpha^{*}$ optimizes the estimation objective function asymptotically. Assumption 4(i.b) delivers the identification by ensuring that $\alpha^{*}$ is the only optimizer. In stating the identification condition, we define $\delta_{i, j, t}\left(\alpha, \alpha^{*}\right):=q_{i, j, t}(\cdot, \alpha)-q_{i, j, t}\left(\cdot, \alpha^{*}\right)$ and use the norm $\|\alpha\|:=\max _{s=1, \ldots, \ell}\left|\alpha_{s}\right|$, where for convenience we also write $\alpha=\left(\alpha_{1}, \ldots, \alpha_{\ell}\right)^{\prime}$.

Assumption 4 (i)(a) There exists $\alpha^{*} \in \mathbb{A}$ such that for all $i, j, t$,

$$
q_{i, j, t}\left(\cdot, \alpha^{*}\right)=q_{i, j, t}^{*}
$$


(b) There is a non-empty index set $\mathcal{I} \subseteq\{(1,1), \ldots,(1, p), \ldots,(n, 1), \ldots,(n, p)\}$ such that for each $\epsilon>0$, there exists $\delta_{\epsilon}>0$ such that for all $\alpha \in \mathbb{A}$ with $\left\|\alpha-\alpha^{*}\right\|>\epsilon$,

$$
P\left[\cup_{(i, j) \in \mathcal{I}}\left\{\left|\delta_{i, j, t}\left(\alpha, \alpha^{*}\right)\right|>\delta_{\epsilon}\right\}\right]>0 .
$$

Among other things, this identification condition ensures that there is sufficient variation in the shape of the conditional distribution to support the estimation of a sufficient number $(\# \mathcal{I})$ of the variation-free conditional quantiles. As in the case of MQ-CAViaR, distributions that depend on a given finite number of variation-free parameters, say $r$, will generally be able to support $r$ variation-free quantiles. For example, the quantiles of the $N(\mu, 1)$ distribution all depend on $\mu$ alone, so there is only one "degree of freedom" for the quantile variation. Similarly, the quantiles of the scaled and shifted $t$-distributions depend on three parameters (location, scale, and kurtosis), so there are only three "degrees of freedom" for the quantile variation.

\section{Asymptotic Theory}

We estimate $\alpha^{*}$ by the quasi-maximum likelihood method. Specifically, we construct a quasi-maximum likelihood estimator (QMLE) $\hat{\alpha}_{T}$ as the solution to the optimization problem

$$
\min _{\alpha \in \mathbb{A}} \bar{S}_{T}(\alpha):=T^{-1} \sum_{t=1}^{T}\left\{\sum_{i=1}^{n} \sum_{j=1}^{p} \rho_{\theta_{i j}}\left(Y_{i t}-q_{i, j, t}(\cdot, \alpha)\right)\right\},
$$

where $\rho_{\theta}(e)=e \psi_{\theta}(e)$ is the standard "check function," defined using the usual quantile step function, $\psi_{\theta}(e)=\theta-1_{[e \leq 0]}$.

We thus view

$$
S_{t}(\alpha):=-\left\{\sum_{i=1}^{n} \sum_{j=1}^{p} \rho_{\theta_{i j}}\left(Y_{i t}-q_{i, j, t}(\cdot, \alpha)\right)\right\}
$$

as the quasi log-likelihood for the observation $t$. In particular, $S_{t}(\alpha)$ is the log-likelihood of a vector of $n p$ independent asymmetric double exponential random variables (see White, 1994, ch. 5.3; Kim and White, 2003; Komunjer, 2005). Because $Y_{i t}-q_{i, j, t}(\cdot, \alpha)$ does not need to actually have this distribution, the method can be regarded as a quasi maximum likelihood.

We establish consistency and asymptotic normality for $\hat{\alpha}_{T}$ through methods analogous to those of White, Kim, and Manganelli (2008). For conciseness, we place the remaining regularity conditions (i.e., Assumptions 5,6 and 7) and technical discussions in the appendix. 
Theorem 1 Suppose that Assumptions 1, 2(i,ii), 3(i), 4(i) and 5(i,ii) old. Then, we have

$$
\hat{\alpha}_{T} \stackrel{a . s .}{\rightarrow} \alpha^{*} .
$$

With $Q^{*}$ and $V^{*}$ as given below, the asymptotic normality result is provided in the following theorem.

Theorem 2 Suppose that Assumptions 1-6 hold. Then, the asymptotic distribution of the QMLE estimator $\hat{\alpha}_{T}$ obtain from (6) is given by:

$$
T^{1 / 2}\left(\hat{\alpha}_{T}-\alpha^{*}\right) \stackrel{d}{\rightarrow} N\left(0, Q^{*-1} V^{*} Q^{*-1}\right),
$$

where

$$
\begin{aligned}
Q^{*}: & =\sum_{i=1}^{n} \sum_{j=1}^{p} E\left[f_{i, j, t}(0) \nabla q_{i, j, t}\left(\cdot, \alpha^{*}\right) \nabla^{\prime} q_{i, j, t}\left(\cdot, \alpha^{*}\right)\right], \\
V^{*}: & =E\left(\eta_{t}^{*} \eta_{t}^{* \prime}\right) \\
\eta_{t}^{*}: & =\sum_{i=1}^{n} \sum_{j=1}^{p} \nabla q_{i, j, t}\left(\cdot, \alpha^{*}\right) \psi_{\theta_{i j}}\left(\varepsilon_{i, j, t}\right), \\
\varepsilon_{i, j, t} \quad: & =Y_{i t}-q_{i, j, t}\left(\cdot, \alpha^{*}\right)
\end{aligned}
$$

We note that the transformed error term of $\psi_{\theta_{i j}}\left(\varepsilon_{i, j, t}\right)=\theta_{i j}-1_{\left[\varepsilon_{i, j, t} \leq 0\right]}$ appearing in Theorem 2 can be viewed as a generalized residual. To test restrictions on $\alpha^{*}$ or to obtain confidence intervals, we require a consistent estimator of the asymptotic covariance matrix $C^{*}:=Q^{*-1} V^{*} Q^{*-1}$. First, we provide a consistent estimator $\hat{V}_{T}$ for $V^{*}$; then we propose a consistent estimator $\hat{Q}_{T}$ for $Q^{*}$. Once $\hat{V}_{T}$ and $\hat{Q}_{T}$ are proved to be consistent for $V^{*}$ and $Q^{*}$ respectively, then it follows by the continuous mapping theorem that $\hat{C}_{T}:=\hat{Q}_{T}^{-1} \hat{V}_{T} \hat{Q}_{T}^{-1}$ is a consistent estimator for $C^{*}$.

A straightforward plug-in estimator of $V^{*}$ is constructed as follows:

$$
\begin{aligned}
\hat{V}_{T} & :=T^{-1} \sum_{t=1}^{T} \hat{\eta}_{t} \hat{\eta}_{t}^{\prime}, \\
\hat{\eta}_{t} & :=\sum_{i=1}^{n} \sum_{j=1}^{p} \nabla q_{i, j, t}\left(\cdot, \hat{\alpha}_{T}\right) \psi_{\theta_{i j}}\left(\hat{\varepsilon}_{i, j, t}\right), \\
\hat{\varepsilon}_{i, j, t} & :=Y_{i t}-q_{i, j, t}\left(\cdot, \hat{\alpha}_{T}\right) .
\end{aligned}
$$

The next result establishes the consistency of $\hat{V}_{T}$ for $V^{*}$.

Theorem 3 Suppose that Assumptions 1-6 hold. Then, we have the following result:

$$
\hat{V}_{T} \stackrel{p}{\rightarrow} V^{*}
$$


Next, we provide a consistent estimator of $Q^{*}$. We follow Powell's (1984) suggestion of estimating $f_{i, j, t}(0)$ with $1_{\left[-\hat{c}_{T} \leq \hat{\varepsilon}_{i, j, t} \leq \hat{c}_{T}\right]} / 2 \hat{c}_{T}$ for a suitably chosen sequence $\left\{\hat{c}_{T}\right\}$. This is also the approach taken in Kim and White (2003), Engle and Manganelli (2004), and White, Kim, and Manganelli (2008). Accordingly, our proposed estimator is

$$
\hat{Q}_{T}=\left(2 \hat{c}_{T} T\right)^{-1} \sum_{i=1}^{n} \sum_{t=1}^{T} \sum_{j=1}^{p} 1_{\left[-\hat{c}_{T} \leq \hat{\varepsilon}_{i, j, t} \leq \hat{c}_{T}\right]} \nabla q_{i, j, t}\left(\cdot, \hat{\alpha}_{T}\right) \nabla^{\prime} q_{i, j, t}\left(\cdot, \hat{\alpha}_{T}\right) .
$$

Theorem 4 Suppose that Assumptions 1-7 hold. Then, we obtain the consistency result for $\hat{Q}_{T}$ as follows:

$$
\hat{Q}_{T} \stackrel{p}{\rightarrow} Q^{*} .
$$

There is no guarantee that $\hat{\alpha}_{T}$ is asymptotically efficient. There is now considerable literature that investigates the efficient estimation in quantile models; see, for example, Newey and Powell (1990), Otsu (2003), Komunjer and Vuong (2006, 2007a, 2007b). Thus far, this literature has only considered single quantile models. It is not obvious how the results for the single quantile models extend to multivariate and multi-quantile models. Nevertheless, Komunjer and Vuong (2007a) show that the class of QML estimators is not large enough to include an efficient estimator, and that the class of $M$-estimators, which strictly includes the QMLE class, yields an estimator that attains the efficiency bound. Specifically, when $p=n=1$, they show that replacing the usual quantile check function $\rho_{\theta_{i j}}(\cdot)$ in equation (6) with

$$
\rho_{\theta_{i j}}^{*}\left(Y_{i t}-q_{i, j, t}(\cdot, \alpha)\right)=\left(\theta_{i j}-1_{\left[Y_{i t}-q_{i, j, t}(\cdot, \alpha) \leq 0\right]}\right)\left(F_{i t}\left(Y_{i t}\right)-F_{i t}\left(q_{i, j, t}(\cdot, \alpha)\right)\right)
$$

will deliver an asymptotically efficient quantile estimator. We conjecture that replacing $\rho_{\theta_{i j}}$ with $\rho_{\theta_{i j}}^{*}$ in equation in (6) will improve the estimator efficiency for $p$ and/or $n$ greater than 1 . We leave the study of the asymptotically efficient multivariate and multi-quantile estimator for future work.

\section{Assessing Tail Reactions of Financial Institu- tions to System Wide Shocks}

The financial crisis which started in 2007 has had a deep impact on the conceptual thinking of systemic risk among both academics and policy makers. There has been a recognition of the shortcomings of the measures that are tailored to dealing with institution-level risks. In particular, institution-level Value at Risk measures miss important externalities associated with the need 
to bail out systemically important banks in order to contain potentially devastating spillovers to the rest of the economy. Therefore, government and supervisory authorities may find themselves compelled to save ex post systemically important financial institutions, while these ignore ex ante any negative externalities associated with their behavior. There exists many contributions, both theoretical and empirical, as summarised, for instance, in Brunnermeier (2012) or Bisias et al. (2012). For the purpose of the application we have in mind, it is useful to structure the material around two early contributions, the CoVaR of Adrian and Brunnermeier (2009) and the systemic expected shortfall (SES) of Acharya et al. (2010).

Both measures aim to capture the risk of a financial institution conditional on a significant negative shock hitting another financial institution or the whole financial system. Formally, the $\operatorname{CoV} R_{\theta}^{j \mid i}$ is the VaR of financial institution $j$ conditional on the event $C$ that hits the financial institution $i$ (denoted by $C^{i}$ ):

$$
\operatorname{Pr}\left(y^{j}<\operatorname{CoVaR}_{\theta}^{j \mid i} \mid C^{i}\right)=\theta .
$$

The systemic expected shortfall is shown to be proportional to the marginal expected shortfall, which is analogously defined as:

$$
M E S_{\theta}^{j \mid i}=E\left(y^{j} \mid C^{i}\right) .
$$

The main difference is that the expectation of the whole left tail, rather than just the quantile, is considered. In practice, loss distributions in the tail are extremely hard to estimate. One strategy is to standardize the returns by the estimated volatility or quantiles, and then apply non-parametric techniques, as done for instance in Manganelli and Engle (2002) or Brownlees and Engle (2010). An alternative is to use extreme value theory to impose a parametric structure on the tail behavior, as done in Hartmann et al. (2004).

As we will show in the rest of this section, the theoretical framework developed in this paper lends itself to a coherent modeling of the dynamics of the tail interdependence implicit in both the CoVaR and systemic expected shortfall measures. Unlike standard GARCH based approaches, which require the modeling of the entire multivariate distribution, the advantage of our multivariate regression quantiles framework - besides providing a robust, semi-parametric technique which does not rely on strong distributional assumptions - is that it is tailored to directly model the object of interest.

In this section, we apply our model to study the spillover that occur in the equity return quantiles of a sample of 230 financial institution around the world. We first describe our empirical model and show how to compute impulse-response functions within the multivariate and multi-quantile framework. We next present the data and the optimization strategy. Finally, we discuss the empirical findings. 


\subsection{Empirical specification}

The specification we use in our empirical analysis is the following simple bivariate quantile model:

$$
q_{t}=c+A\left|Y_{t-1}\right|+B q_{t-1},
$$

where $q_{t}, Y_{t-1}$, and $c$ are 2 -dimensional vectors, and $A, B$ are $(2,2)$-matrices. The parameters can be consistently estimated by minimizing the multivariate regression quantile objective function (6). It is straightforward to derive an estimate of the CoVaR from this model. For instance, if the conditioning event $C^{i}$ is defined as $Y_{2, t-1}=q_{2, t-1}$, that is financial institution 2 is hit by a shock equal to its quantile, the associated CoVaR for financial institution 1 is given by $q_{1, t}=c_{1}+a_{11}\left|Y_{1 t-1}\right|+a_{12}\left|q_{2, t-1}\right|+b_{11} q_{1, t-1}+b_{12} q_{2, t-1} \cdot{ }^{1}$

A DGP consistent with (7) can be obtained assuming that the data are structurally generated as:

$$
Y_{t}=L_{t} \varepsilon_{t},
$$

where $L_{t}:=L_{t}\left(Z^{t-1}\right)$ is an $\mathcal{F}_{t}-$ measurable lower triangular matrix and the elements of $\varepsilon_{t}:=\left[\varepsilon_{1 t}, \varepsilon_{2 t}\right]^{\prime}$ are mutually independent with $\left\{\varepsilon_{t} \mid \mathcal{F}_{t}\right\}$ being a martingale difference sequence.

A suitable choice of $L_{t}$ ensuring that the conditional quantiles of $Y_{t}$ obey (4) and (7) is the following bivariate model:

$$
\left[\begin{array}{l}
Y_{1 t} \\
Y_{2 t}
\end{array}\right]=\left[\begin{array}{cc}
\alpha_{t} & 0 \\
\beta_{t} & \gamma_{t}
\end{array}\right]\left[\begin{array}{l}
\varepsilon_{1 t} \\
\varepsilon_{2 t}
\end{array}\right]
$$

where $\varepsilon_{t}$ is the bivariate standard normal random variable. Note that the standard deviations of $Y_{1 t}$ and $Y_{2 t}$ are given by $\sigma_{t}\left(Y_{1 t}\right)=\alpha_{t}$ and $\sigma_{t}\left(Y_{2 t}\right)=$ $\sqrt{\beta_{t}^{2}+\gamma_{t}^{2}}$ respectively. Suppose that $\alpha_{t}, \beta_{t}$ and $\gamma_{t}$ are specified in such a way to satisfy the following:

$$
\begin{aligned}
& \sigma_{t}\left(Y_{1 t}\right)=\tilde{c}_{1}+\tilde{a}_{11}\left|Y_{1 t-1}\right|+\tilde{a}_{12}\left|Y_{2 t-1}\right|+b_{11} \sigma_{1 t-1}+b_{12} \sigma_{2 t-1} \\
& \sigma_{t}\left(Y_{2 t}\right)=\tilde{c}_{2}+\tilde{a}_{21}\left|Y_{1 t-1}\right|+\tilde{a}_{22}\left|Y_{2 t-1}\right|+b_{21} \sigma_{1 t-1}+b_{22} \sigma_{2 t-1}
\end{aligned}
$$

The respective quantile processes associated with this DGP are given by:

$$
\begin{aligned}
& q_{1 t}=k \tilde{c}_{1}+k \tilde{a}_{11}\left|Y_{1 t-1}\right|+k \tilde{a}_{12}\left|Y_{2 t-1}\right|+b_{11} q_{1 t-1}+b_{12} q_{2 t-1}, \\
& q_{2 t}=k \tilde{c}_{2}+k \tilde{a}_{21}\left|Y_{1 t-1}\right|+k \tilde{a}_{22}\left|Y_{2 t-1}\right|+b_{21} q_{1 t-1}+b_{22} q_{2 t-1},
\end{aligned}
$$

where $k$ is the $\theta^{\text {th }}$-quantile of the standard normal distribution. In matrix form, the above system can be rewritten as in (7), where $c_{i}=k \tilde{c}_{i}$ and $a_{i j}=k \tilde{a}_{i j}$.

\footnotetext{
${ }^{1} Y_{i, t}$ denotes the i-th element of the vector $Y_{t}$, while $a_{i j}$ is the element of the i-th row and j-th column of the matrix $A$. Similar notation is used to denote the individual elements of the vectors and matrices $q_{t}, c$ and $B$.
} 
In the empirical application, we impose the following identification assumption:

Identification Assumption: The first element of $Y_{t}$ in (8) denotes the per-period return on a financial index and the second element is the per-period return on a specific financial institution within the index.

The identification assumption behind this decomposition is that shocks to the financial index are allowed to have a direct impact on the return of the specific financial institution, but shocks to the specific financial institution do not have a direct impact on the financial index. Here, we limit ourselves to a bivariate system, as we are interested in the interaction between a financial index and an individual financial institution. The theoretical framework of this paper can accommodate higher dimensional models, although at the cost of increasing the computational burden.

Incidentally, this identification scheme illustrates the potential pitfalls of choosing appropriate conditioning events for the CoVaR measures. Defining the conditioning event $C^{i}$ as $Y_{2, t-1}=q_{2, t-1}$, as done before, neglects the fact that shock to the financial institution 2 may be correlated with that of other financial institutions, therefore producing a potentially misleading classification of the systemic importance of financial institutions. In the following, based on the identification scheme which is implicit in the triangular structure of $L_{t}$, our attention will focus mainly on the impact of market shocks on the VaR of individual financial institutions.

\subsubsection{The Quantile Impuse Response Function (QIRF)}

This modeling framework allows us to proceed a step further beyond the scope of the static analysis implicit in the CoVaR framework because we can introduce the concept of impulse-response functions for quantiles. To see how quantile impulse-response functions can be defined, assume that in the DGP (8) there is a shock $\delta$ (or intervention) to $\varepsilon_{1 t}$ only at time $t$ so that $\tilde{\varepsilon}_{1 t} \equiv \varepsilon_{1 t}+\delta$ (since $\varepsilon_{t}$ is assumed to be the standard normal, it is actually $\delta$-standard deviation). In all other times there is no intervention. In other words, the time path of the error terms without the intervention would be

$$
\left\{\ldots, \varepsilon_{1 t-2}, \varepsilon_{1 t-1}, \varepsilon_{1 t}, \varepsilon_{1 t+1}, \varepsilon_{1 t+2}, \ldots\right\}
$$

while the time path with the intervention would be

$$
\left\{\ldots, \varepsilon_{1 t-2}, \varepsilon_{1 t-1}, \tilde{\varepsilon}_{1 t}, \varepsilon_{1 t+1}, \varepsilon_{1 t+2}, \ldots\right\} .
$$

Our objective is the measure the impact of the one-off intervention at time $t$ on the quantile dynamics.

We first consider the time path of $Y_{t}$ without and with the intervention. The affected $Y_{t}$ will be denoted as $\tilde{Y}_{t}$. Note that the intervention $\delta$ at time 
$t$ will only affect $Y_{t}$, but not the future realizations of $Y_{t+s}(s=1,2,3, \ldots)$, due to the way we specify our model. Hence, the time path of $\tilde{Y}_{t}$ with the intervention is given by

$$
\left\{\ldots, Y_{t-2}, Y_{t-1}, \tilde{Y}_{t}, Y_{t+1}, Y_{t+2}, \ldots\right\} .
$$

In fact, the difference between the origainal series and the affected series is zero for $n \geq 1$. Furthermore note that $\tilde{Y}_{t}=L_{t}\left[\tilde{\varepsilon}_{1 t}, \varepsilon_{2 t}\right]^{\prime}$ because of (8).

The $\theta^{\text {th }}$ quantile impulse-response function (QIRF) for the $i^{\text {th }}$ variable $\left(Y_{i t}\right)$ denoted as $\Delta_{i, s}\left(\tilde{\varepsilon}_{1 t}\right)$ is defined as

$$
\Delta_{i, s}\left(\tilde{\varepsilon}_{1 t}\right)=\tilde{q}_{i, t+s}-q_{i, t+s} \quad s=1,2,3, \ldots
$$

where $\tilde{q}_{i, t+s}$ is the $\theta^{t h}$ conditional quantile of the affected series $\left(\tilde{Y}_{i t+s}\right)$ and $q_{i, t+s}$ is the $\theta^{t h}$ conditional quantile of the unaffected series $\left(Y_{i t+s}\right)$.

First, we consider the case for $i=1$, i.e. $\Delta_{1, s}\left(\tilde{\varepsilon}_{1 t}\right)$. When $s=1$, the QIRF is given by

$$
\Delta_{1,1}\left(\tilde{\varepsilon}_{1 t}\right)=a_{11}\left(\left|\tilde{Y}_{1 t}\right|-\left|Y_{1 t}\right|\right)+a_{12}\left(\left|\tilde{Y}_{2 t}\right|-\left|Y_{2 t}\right|\right) .
$$

For $s>1$, the QIRF is given by

$$
\Delta_{1, s}\left(\tilde{\varepsilon}_{1 t}\right)=b_{11} \Delta_{1, s-1}\left(\tilde{\varepsilon}_{1 t}\right)+b_{12} \Delta_{2, s-1}\left(\tilde{\varepsilon}_{1 t}\right) .
$$

The case for $i=2$ is similarly obtained as follows. For $s=1$,

$$
\Delta_{2,1}\left(\tilde{\varepsilon}_{1 t}\right)=a_{21}\left(\left|\tilde{Y}_{1 t}\right|-\left|Y_{1 t}\right|\right)+a_{22}\left(\left|\tilde{Y}_{2 t}\right|-\left|Y_{2 t}\right|\right),
$$

while for $s>1$,

$$
\Delta_{2, s}\left(\tilde{\varepsilon}_{1 t}\right)=b_{21} \Delta_{1, s-1}\left(\tilde{\varepsilon}_{1 t}\right)+b_{22} \Delta_{2, s-1}\left(\tilde{\varepsilon}_{1 t}\right) .
$$

Now, let us define

$$
\Delta_{s}\left(\tilde{\varepsilon}_{1 t}\right):=\left[\begin{array}{c}
\Delta_{1, s}\left(\tilde{\varepsilon}_{1 t}\right) \\
\Delta_{2, s}\left(\tilde{\varepsilon}_{1 t}\right)
\end{array}\right],
$$

and

$$
\begin{aligned}
D_{t} & =\left|\tilde{Y}_{t}\right|-\left|Y_{t}\right| \\
& =\left|L_{t} \tilde{\varepsilon}_{t}^{1}\right|-\left|L_{t} \varepsilon_{t}\right| .
\end{aligned}
$$

where $\tilde{\varepsilon}_{t}^{1}=\left[\tilde{\varepsilon}_{1 t}, \varepsilon_{2 t}\right]^{\prime}$. Then, we can show that the QIRF is compactly expressed as follows:

$$
\begin{aligned}
& \Delta_{s}\left(\tilde{\varepsilon}_{1 t}\right)=A D_{t} \\
& \Delta_{s}\left(\tilde{\varepsilon}_{1 t}\right)=B^{(s-1)} A D_{t} \quad \text { for } s>1 .
\end{aligned}
$$

The QIRF when there is a shock (or intervention) to $\varepsilon_{2 t}$ only at time $t$ can be analogously obtained.

In the empirical application, the matrix $L_{t}$ - needed to compute the impact of $\tilde{\varepsilon}_{1 t}$ on $Y_{t}$ - is estimated using a standard Cholesky decomposition. 


\subsection{Data and Optimization Strategy}

The data used in this section have been downloaded from Datastream. We considered three main global sub-indices: banks, financial services, and insurances. The sample includes daily closing prices from 1 January 2000 to 6 August 2010. Prices were transformed into continuously compounded log returns, giving an estimation sample size of 2765 observations. We use 453 additional observations up to 2 May 2012, for the out-of-sample exercises. We eliminated all the stocks whose times series started later than 1 January 2000 , or which stopped after this date. At the end of this process, we were left with 230 stocks.

Table 1 reports the names of the financial institutions in our sample, together with the country of origin and the sector they are associated with, as from Datastream classification. Table 2 shows the breakdown of the stocks by sector and by geographic area. There are twice as many financial institutions classified as banks in our sample relative to those classified as financial services or insurances. The distribution across geographic areas is more balanced, with a greater number of EU financial institutions and a slightly lower Asian representation. The proxy for the market index used in each bivariate quantile estimation is the equally weighted average of all the financial institutions in the same geographic area, in order to avoid asynchronicity issues.

We estimated 230 bivariate $1 \%$ quantile models between the market index and each of the 230 financial institutions in our sample. Each model is estimated using, as starting values in the optimization routine, the univariate CAViaR estimates and initializing the remaining parameters at zero. We also generated 40 additional initial conditions by adding a normally distributed noise to this vector. For each of these 40 initial conditions, we minimized the regression quantile objective function (6) using the fminsearch optimization function in Matlab, which is based on the Nelder-Mead simplex algorithm. Finally, among the resulting 40 vectors of the parameter estimates, we chose the vector yielding the lowest value for the function (6). We adopt this strategy because we have found that parameter estimates are sometimes sensitive to the choices of the initial conditions (possibly due to a flat likelihood near the optimum). Such an optimization strategy is more time consuming, but delivers more reliable results. In calculating the standard errors, we have set the bandwidth to 1 throughout the sample.

\subsection{Results}

Table 3 reports, as an example, the estimation results for four well-known financial institutions: Barclays, Deutsche Bank, Citigroup and Goldman Sachs. The diagonal autoregressive coefficients for the $B$ matrix are around 0.90 and all of them are statistically significant, which indicates the VaR 
processess are significantly autocorrelated. These findings are consistent with what is typically found in the literature using CAViaR models. Notice, however, that some of the non-diagonal coefficients for the $A$ and $B$ matrices are significantly different from zero, illustrating how the multivariate quantile model can uncover dynamics that cannot be detected by estimating univariate quantile models. In general, we reject the joint null hypothesis that all off-diagonal coefficients of the matrices $A$ and $B$ are equal to zero at the $5 \%$ level for 142 financial institutions out of the 230 in our sample. The resulting estimated 1\% quantiles for Barclays, Deutsche Bank, Citigroup and Goldman Sachs are reported in Figure 1. The quantile plots clearly reveal the generalized sharp increase in risk following the Lehman bankruptcy. Careful inspection of the plots also reveals a noticeable cross-sectional difference, with the risk for Goldman Sachs being contained to less than half the risk of Citigroup at the height of the crisis.

The methodology introduced in this paper, however, allows us to go beyond the analysis of the univariate quantiles, and directly looks at the tail codependence between financial institutions and the market index. Figure 2 displays the impulse response of the risks of the four financial institutions to a 2 standard deviation shock to the market index (see the discussion in the previous sub-section for a detailed explanation of how the impulse-response functions are computed). The horizontal axis measures the time (expressed in days), while the vertical axis measures the change in the $1 \%$ quantiles of the individual financial institutions (expressed in percentage returns) as a reaction to the market shock. The impulse response functions track how this shock propagates through the system and how long it takes to absorb it. The shock is completely reabsorbed after the impulse response function has converged again to zero.

Looking more closely at the impulse response functions of the four selected financial institutions reveals a few differences in how their long run risks react to shocks. For instance, Deutsche Bank and Barclays have a sharp initial reaction. However, while the shock to Deutsche Bank's VaR is entirely absorbed after around 35 days, the shock to Barclays' risk appear to be more persistent, with its effect not being completely absorbed after more than 50 days. Similarly, risk shocks on Citigroup's VaR appears to be long lasting, while Goldman Sachs quantiles overall exhibit very little tail correlation with the market.

It should be borne in mind that each of the 230 bivariate models is estimated using a different information set (as the time series of the index and of a different financial institution is used for each estimation). Therefore, each pair produces a different estimate of the VaR of the index, simply because we condition on a different information set. Moreover, the coefficients and any quantities derived from them, such as impulse responses, are information set-specific. This means that naive comparisons across bivariate pairs can be misleading and are generally unwarranted. The proper context 
for comparing sensitivities and impulse responses is in a multivariate setting using a common information set. Because of the non trivial computational challenges involved, we leave this for future study.

Nevertheless, averaging across the bivariate results can still provide useful summary information and suggest general features of the results. Accordingly, Figure 3 plots the average impulse-response functions $\Delta_{1, s}\left(\tilde{\varepsilon}_{2 t}\right)$ and $\Delta_{2, s}\left(\tilde{\varepsilon}_{1 t}\right)$ measuring the impact of a two standard deviation individual financial institution shock on the index and the impact of a two standard deviation shock to the index on the individual financial institution's risk. In the left column, the average is taken with respect to the geographical distribution. That is, the average impulse-response for Europe, for example, is obtained by averaging all the impulse-response functions for the European financial institutions. We notice two things. First, the impact of a shock to the index (charts in the top row) is much stronger than the impact of a shock to the individual financial institution (charts in the bottom row). This result is partly driven by our identification assumption that shocks to the index have a contemporaneous impact on the return of the single financial institutions, while the institution's specific shocks have only a lagged impact on the global financial index. Second, we notice that the risk of Asian financial institutions appears to be on average somewhat less sensitive to global shocks than their European and North American counterparts.

The charts on the right column of Figure 3 plot the average impulseresponse functions for the financial institutions grouped by line of business, i.e. banks, financial services, and insurances. We see that a shock to the index has a stronger initial impact on the group of insurance companies. Regarding the impact of shocks to the individual financial institutions on the risk of the global index, banks have on average a lower initial impact, but the shock appears to be more persistent than for financial services and insurance companies.

Overall, however, it is fair to say that no big differences can be noted among the impulse responses by aggregating over the geographic or sectorial dimension. To highlight the still sizeable cross-sectional difference, and to get an idea of the orders of magnitude involved, we ranked the financial institutions by their overall risk impact by integrating out all the individual impulse-responses. Figure 4 plots the average impulse-responses which correspond to the 20 financial institutions whose risk is most and least sensitive to market shocks, together with those of the largest and smallest impact on the risk of the market risk index. It is clear that the shocks to the index have an impact of an order of magnitude greater than that of the shocks to the individual financial institutions. A two standard deviation shock to the index produces an average initial increase in the daily $\mathrm{VaR}$ of the most sensitive financial institutions at more than $3 \%$. The shock is also quite persistent, as it is not yet completely absorbed after 50 days. On the other hand, for the least sensitive financial institutions, a shock to the index pro- 
duces an average immediate increase in the VaR of less than $1 \%$, which is then entirely absorbed after the third week.

To gauge what extent the model correctly identifies the financial institutions whose risks are most exposed to market shocks, Figure 5 plots the average quantiles of the two sets of financial institutions identified in Figure 4. Specifically, the charts in the top panels of the figure, track the estimated in-sample quantiles and stock price developments of the 20 financial institutions which have been identified in Figure 4 as being most and least exposed to market shocks. For comparison, we have added the risk and price developments of the market index. Prices have been normalized to 100 at the beginning of the sample. The charts in the bottom panels replicate the same exercise with the out-of-sample data.

The figure presents two striking facts. First, during normal times, i.e. between 2004 and mid-2007, the quantiles of the most and least sensitive groups of financial institutions is roughly equal. Actually, there are some periods in 2003 in which the quantiles of the least sensitive financial institutions exceeded the quantiles of the most sensitive ones. The second striking fact is that the situation changes abruptly in periods of market turbulence. For instance, at the beginning of the sample, in 2001-2003, the quantiles of the most sensitive financial institutions increased significantly more than that of the least sensitive ones. The change in behavior during crisis periods is even more striking from 2008 onwards, showing a greater exposure to common shocks. The chart on price developments on the right hand side confirms that the group of financial institutions identified as the most sensitive to market shocks are those whose stock market value dropped the most during the Lehman crisis, with their values dropping on 18 February 2009 by more than $90 \%$ with respect to the beginning of the sample. In contrast, the values of the least sensitive financial institutions have remained relatively stable throughout the sample, and in particular, they suffer only minor losses at the height of the crisis. The bottom panels reveal that the same result holds for the out-of-sample period. Of particular notice is the sharp drop in the out-of-sample quantile for the group of the most sensitive financial institutions which occurred on 12 August, 2011, the beginning of the second phase of the euro area sovereign debt crisis.

This application illustrates how the proposed methodology can usefully inform policy makers by helping identify the set of financial institutions which may be most exposed to common shocks, especially in times of crisis. Of course, this should only be considered as a partial model-based screening device for the identification of the most systemic banks. Further analysis, market intelligence and sound judgment are other necessary elements to produce a reliable risk assessment method for the larger and more complex financial groups.

Again, we emphasize that the results presented in these figures merely summarize the pattern of the results found in the bivariate analysis of our 
230 financial institutions. Cross-comparisons could be improved by estimating for instance a 3 - or 4- or $n$-variate system using a common information set, or adopting an appropriate factor structure which would minimize the number of parameters to be estimated. Alternatively, one could impose that the $B$ matrix in (7) is diagonal, which would be equivalent to assuming that the parameters of the system are variation free, as in Engle et al. (1983). This assumption would have the added advantage of allowing a separate estimation of each quantile. That is, for an $n$-variate system, the optimization problem in (6) can be broken down into $n$ independent optimization problems, which in turn would considerably increase the computational tractability.

\section{Conclusion}

We have developed a theory ensuring the consistency and asymptotic normality of multivariate and multi-quantile models. Our theory is general enough to comprehensively cover models with multiple random variables, multiple confidence levels and multiple lags of the quantiles.

We conducted an empirical analysis in which we estimate a vector autoregressive model for the Value at Risk - VAR for VaR - using returns of individual financial institutions from around the world and a global financial sector index. By examining the impulse-response functions, we can study the financial institutions' long run risk reactions to shocks to the overall index. Judging from our bivariate models, we found that the risk of Asian financial institutions tend to be less sensitive to system wide shocks, whereas insurance companies exhibit a greater sensitivity to global shocks. By looking at the integral of all the individual impulse-responses, we found wide differences on how financial institutions react to different shocks. Both in-sample and out-of-sample analyses reveal that financial institutions with the strongest impulse-responses to global shocks are those which suffer the most in periods of market turbulence.

The methods developed in this paper can be applied to many other contexts. For instance, many stress-test models are built from vector autoregressive models on credit risk indicators and macroeconomic variables. Starting from the estimated mean and adding assumptions on the multivariate distribution of the error terms, one can deduce the impact of a macro shock on the quantile of the credit risk variables. Our methodology provides a convenient alternative for stress testing, by allowing researchers to estimate vector autoregressive processes directly on the quantiles of interest, rather than on the mean. 


\section{References}

[1] Acharya, V., Pedersen, L., Philippe, T., and Richardson, M. (2010). Measuring Systemic Risk. Technical report, Department of Finance, NYU.

[2] Adrian, T. and Brunnermeier, M. (2009). CoVaR. Manuscript, Princeton University.

[3] Andersen, T. G., Bollerslev, T., Diebold, F. X., and Labys, P. (2003). Modeling and Forecasting Realized Volatility. Econometrica 71, 579625 .

[4] Andrews, D.W.K. (1988). Laws of Large Numbers for Dependent NonIdentically Distributed Random Variables. Econometric Theory 4, 458467.

[5] Bartle, R. (1966). The Elements of Integration. New York: Wiley.

[6] Bisias, D., Flood, M., Lo, A.W., and Valavanis, S. (2012). A Survey of Systemic Risk Analyics. Office of Financial Research Working Paper.

[7] Brownlees, C.T. and Engle, R.F. (2011). Volatility, Correlation and Tails for Systemic Risk Measurement. Manuscript, Stern School of Business, New York University.

[8] Brunnermeier, M. and Oehmke, M. (2012). Bubbles, Financial Crises, and Systemic Risk. Handbook of Finance and Economics, forthcoming.

[9] Engle, R.F., Hendry, D.F., and Richard, J.-F. (1983). Exogeneity. Econometrica 51, 277-304.

[10] Engle, R.F. and Manganelli, S. (2004). CAViaR: Conditional Autoregressive Value at Risk by Regression Quantiles. Journal of Business 8 Economic Statistics 22, 367-381.

[11] Hartmann, P., Straetmans, S., and de Vries, C.G. (2004). Asset Market Linkages in Crisis Periods. Review of Economics and Statistics 86, 313326.

[12] Huber, P.J. (1967). The Behavior of Maximum Likelihood Estimates under Nonstandard Conditions. Proceedings of the Fifth Berkeley Symposium on Mathematical Statistics and Probability. Berkeley: University of California Press, pp. 221-233.

[13] Kim, T.H. and White, H. (2003). Estimation, Inference, and Specification Testing for Possibly Misspecified Quantile Regression. In T. Fomby and C. Hill, eds., Maximum Likelihood Estimation of Misspecified Models: Twenty Years Later. New York: Elsevier, pp. 107-132. 
[14] Koenker, R. and Bassett, G. (1978). Regression Quantiles. Econometrica $46,33-50$.

[15] Komunjer, I. (2005). Quasi-Maximum Likelihood Estimation for Conditional Quantiles. Journal of Econometrics 128, 127-164.

[16] Komunjer, I. and Vuong, Q. (2006). Efficient Conditional Quantile Estimation: the Time Series Case. University of California, San Diego Department of Economics Discussion Paper 2006-10, .

[17] Komunjer, I. and Vuong, Q. (2007a). Semiparametric Efficiency Bound and M-Estimation in Time-Series Models for Conditional Quantiles. University of California, San Diego Department of Economics Discussion Paper.

[18] Komunjer, I. and Vuong, Q. (2007b). Efficient Estimation in Dynamic Conditional Quantile Models. University of California, San Diego Department of Economics Discussion Paper.

[19] Manganelli, S. and Engle, R.F. (2002). Value at Risk Models in Finance. ECB Working Paper No. 75.

[20] Newey, W.K. and Powell, J.L. (1990). Efficient Estimation of Linear and Type I Censored Regression Models under Conditional Quantile Restrictions. Econometric Theory 6, 295-317.

[21] Otsu, T. (2003). Empirical Likelihood for Quantile Regression. University of Wisconsin, Madison Department of Economics Discussion Paper.

[22] Powell, J. (1984). Least Absolute Deviations Estimators for the Censored Regression Model. Journal of Econometrics 25, 303-325.

[23] Stinchcombe, M. and White, H. (1998). Consistent Specification Testing with Nuisance Parameters Present only under the Alternative. Econometric Theory 14, 295-324.

[24] Weiss, A. (1991). Estimating Nonlinear Dynamic Models Using Least Absolute Error Estimation. Econometric Theory 7, 46-68.

[25] White, H. (1994). Estimation, Inference and Specification Analysis. New York: Cambridge University Press.

[26] White, H. (2001). Asymptotic Theory for Econometricians. San Diego: Academic Press.

[27] White, H. (2006). Approximate Nonlinear Forecasting Methods. In G. Elliott, C.W.J. Granger, and A. Timmermann, eds., Handbook of Economic Forecasting. New York: Elsevier, pp. 460-512. 
[28] White, H., Kim, T.H., and Manganelli, S. (2008). Modeling Autoregressive Conditional Skewness and Kurtosis with Multi-Quantile CAViaR. In J. Russell and M. Watson, eds., Volatility and Time Series Econometrics: A Festschrift in Honor of Robert F. Engle. 


\section{Appendix}

We establish the consistency of $\hat{\alpha}_{T}$ by applying the results of White (1994). For this, we impose the following moment and domination conditions. In stating this next condition and where convenient elsewhere, we exploit stationarity to omit explicit reference to all values of $t$.

Assumption 5 (i) For $i=1, \ldots, n, E\left|Y_{i t}\right|<\infty$; (ii) let $D_{0, t}:=\max _{i=1, \ldots, n} \max _{j=1, \ldots, p}$ $\sup _{\alpha \in \mathbb{A}}\left|q_{i, j, t}(\cdot, \alpha)\right|$. Then $E\left(D_{0, t}\right)<\infty$.

Proof of Theorem 1 We verify the conditions of corollary 5.11 of White (1994), which delivers $\hat{\alpha}_{T} \rightarrow \alpha^{*}$, where

$$
\hat{\alpha}_{T}:=\arg \max _{\alpha \in \mathbb{A}} T^{-1} \sum_{t=1}^{T} \varphi_{t}\left(Y_{t}, q_{t}(\cdot, \alpha)\right),
$$

and $\varphi_{t}\left(Y_{t}, q_{t}(\cdot, \alpha)\right):=-\left\{\sum_{i=1}^{n} \sum_{j=1}^{p} \rho_{\theta_{i j}}\left(Y_{i t}-q_{i, j, t}(\cdot, \alpha)\right)\right\}$. Assumption 1 ensures White's Assumption 2.1. Assumption 3(i) ensures White's Assumption 5.1. Our choice of $\rho_{\theta_{i j}}$ satisfies White's Assumption 5.4. To verify White's Assumption 3.1, it suffices that $\varphi_{t}\left(Y_{t}, q_{t}(\cdot, \alpha)\right)$ is dominated on $\mathbb{A}$ by an integrable function (ensuring White's Assumption 3.1(a,b)), and that for each $\alpha$ in $\mathbb{A},\left\{\varphi_{t}\left(Y_{t}, q_{t}(\cdot, \alpha)\right)\right\}$ is stationary and ergodic (ensuring White's Assumption 3.1(c), the strong uniform law of large numbers (ULLN)). Stationarity and ergodicity is ensured by Assumptions 1 and 3(i). To show domination, we write

$$
\begin{aligned}
\left|\varphi_{t}\left(Y_{t}, q_{t}(\cdot, \alpha)\right)\right| & \leq \sum_{i=1}^{n} \sum_{j=1}^{p}\left|\rho_{\theta_{i j}}\left(Y_{i t}-q_{i, j, t}(\cdot, \alpha)\right)\right| \\
& =\sum_{i=1}^{n} \sum_{j=1}^{p}\left|\left(Y_{i t}-q_{i, j, t}(\cdot, \alpha)\right)\left(\theta_{i j}-1_{\left[Y_{i t}-q_{i, j, t}(\cdot, \alpha) \leq 0\right]}\right)\right| \\
& \leq 2 \sum_{i=1}^{n} \sum_{j=1}^{p}\left(\left|Y_{i t}\right|+\left|q_{i, j, t}(\cdot, \alpha)\right|\right) \\
& \leq 2 p \sum_{i=1}^{n}\left|Y_{i t}\right|+2 n p\left|D_{0, t}\right|
\end{aligned}
$$

so that

$$
\sup _{\alpha \in \mathbb{A}}\left|\varphi_{t}\left(Y_{t}, q_{t}(\cdot, \alpha)\right)\right| \leq 2 p \sum_{i=1}^{n}\left|Y_{i t}\right|+2 n p\left|D_{0, t}\right|
$$

Thus, $2 p \sum_{i=1}^{n}\left|Y_{i t}\right|+2 n p\left|D_{0, t}\right|$ dominates $\left|\varphi_{t}\left(Y_{t}, q_{t}(\cdot, \alpha)\right)\right|$; this has finite expectation by Assumption 5(i,ii). 
White's Assumption 3.2 remains to be verified; here, this is the condition that $\alpha^{*}$ is the unique maximizer of $E\left(\varphi_{t}\left(Y_{t}, q_{t}(\cdot, \alpha)\right)\right.$. Given Assumptions 2(ii.b) and 4(i), it follows through the argument that directly parallels to that of the proof by White (1994, corollary 5.11) that for all $\alpha \in \mathbb{A}$,

$$
E\left(\varphi_{t}\left(Y_{t}, q_{t}(\cdot, \alpha)\right) \leq E\left(\varphi_{t}\left(Y_{t}, q_{t}\left(\cdot, \alpha^{*}\right)\right) .\right.\right.
$$

Thus, it suffices to show that the above inequality is strict for $\alpha \neq \alpha^{*}$. Consider $\alpha \neq \alpha^{*}$ such that $\left\|\alpha-\alpha^{*}\right\|>\epsilon$, and let $\Delta(\alpha):=\sum_{i=1}^{n} \sum_{j=1}^{p} E\left(\Delta_{i, j, t}(\alpha)\right)$ with $\Delta_{i, j, t}(\alpha):=\rho_{\theta_{i j}}\left(Y_{i t}-q_{i, j, t}(\cdot, \alpha)\right)-\rho_{\theta_{i j}}\left(Y_{i t}-q_{i, j, t}\left(\cdot, \alpha^{*}\right)\right)$. It will suffice to show that $\Delta(\alpha)>0$. First, we define the "error" $\varepsilon_{i, j, t}:=Y_{i t}-q_{i, j, t}\left(\cdot, \alpha^{*}\right)$ and let $f_{i, j, t}(\cdot)$ be the density of $\varepsilon_{i, j, t}$ conditional on $\mathcal{F}_{t-1}$. Noting that $\delta_{i, j, t}\left(\alpha, \alpha^{*}\right):=q_{i, j, t}(\cdot, \alpha)-q_{i, j, t}\left(\cdot, \alpha^{*}\right)$, we next can show through some algebra and Assumption 2(ii.a) that

$$
\begin{aligned}
E\left(\Delta_{i, j, t}(\alpha)\right) & =E\left[\int_{0}^{\delta_{i, j, t}\left(\alpha, \alpha^{*}\right)}\left(\delta_{i, j, t}\left(\alpha, \alpha^{*}\right)-s\right) f_{i, j, t}(s) d s\right] \\
& \left.\geq E\left[\frac{1}{2} \delta_{\epsilon}^{2} 1_{\left[\left|\delta_{i, j, t}\left(\alpha, \alpha^{*}\right)\right|>\delta_{\epsilon}\right]}+\frac{1}{2} \delta_{i, j, t}\left(\alpha, \alpha^{*}\right)^{2} 1_{\left[\left|\delta_{i, j, t}\left(\alpha, \alpha^{*}\right)\right| \leq \delta_{\epsilon}\right]}\right)\right] \\
& \geq \frac{1}{2} \delta_{\epsilon}^{2} E\left[1_{\left[\left|\delta_{i, j, t}\left(\alpha, \alpha^{*}\right)\right|>\delta_{\epsilon}\right]}\right] .
\end{aligned}
$$

The first inequality above comes from the fact that Assumption 2(ii.a) implies that for any $\delta>0$ sufficiently small, we have $f_{i, j, t}(s)>\delta$ for $|s|<\delta$. Thus,

$$
\begin{aligned}
\Delta(\alpha) & :=\sum_{i=1}^{n} \sum_{j=1}^{p} E\left(\Delta_{i, j, t}(\alpha)\right) \geq \frac{1}{2} \delta_{\epsilon}^{2} \sum_{i=1}^{n} \sum_{j=1}^{p} E\left[1_{\left[\left|\delta_{i, j, t}\left(\alpha, \alpha^{*}\right)\right|>\delta_{\epsilon}\right]}\right] \\
& =\frac{1}{2} \delta_{\epsilon}^{2} \sum_{i=1}^{n} \sum_{j=1}^{p} P\left[\left|\delta_{i, j, t}\left(\alpha, \alpha^{*}\right)\right|>\delta_{\epsilon}\right] \geq \frac{1}{2} \delta_{\epsilon}^{2} \sum_{(i, j) \in \mathcal{I}} P\left[\left|\delta_{i, j, t}\left(\alpha, \alpha^{*}\right)\right|>\delta_{\epsilon}\right] \\
& \geq \frac{1}{2} \delta_{\epsilon}^{2} P\left[\cup_{(i, j) \in \mathcal{I}}\left\{\left|\delta_{i, j, t}\left(\alpha, \alpha^{*}\right)\right|>\delta_{\epsilon}\right\}\right]>0,
\end{aligned}
$$

where the final inequality follows from Assumption 4(i.b). As $\alpha$ is arbitrary, the result follows.

Next, we establish the asymptotic normality of $T^{1 / 2}\left(\hat{\alpha}_{T}-\alpha^{*}\right)$. We use a method originally proposed by Huber (1967) and later extended by Weiss (1991). We first sketch the method before providing formal conditions and the proof.

Huber's method applies to our estimator $\hat{\alpha}_{T}$, provided that $\hat{\alpha}_{T}$ satisfies the asymptotic first order conditions

$$
T^{-1} \sum_{t=1}^{T}\left\{\sum_{i=1}^{n} \sum_{j=1}^{p} \nabla q_{i, j, t}\left(\cdot, \hat{\alpha}_{T}\right) \psi_{\theta_{i j}}\left(Y_{i t}-q_{i, j, t}\left(\cdot, \hat{\alpha}_{T}\right)\right)\right\}=o_{p}\left(T^{1 / 2}\right),
$$


where $\nabla q_{i, j, t}(\cdot, \alpha)$ is the $\ell \times 1$ gradient vector with elements $\left(\partial / \partial \alpha_{s}\right) q_{i, j, t}(\cdot, \alpha), s=$ $1, \ldots, \ell$, and $\psi_{\theta_{i j}}\left(Y_{i t}-q_{i, j, t}\left(\cdot, \hat{\alpha}_{T}\right)\right)$ is a generalized residual. Our first task is thus to ensure that equation (9) holds.

Next, we define

$$
\lambda(\alpha):=\sum_{i=1}^{n} \sum_{j=1}^{p} E\left[\nabla q_{i, j, t}(\cdot, \alpha) \psi_{\theta_{i j}}\left(Y_{i t}-q_{i, j, t}(\cdot, \alpha)\right)\right] .
$$

With $\lambda(\alpha)$ continuously differentiable at $\alpha^{*}$ interior to $\mathbb{A}$, we can apply the mean value theorem to obtain

$$
\lambda(\alpha)=\lambda\left(\alpha^{*}\right)+Q_{0}\left(\alpha-\alpha^{*}\right),
$$

where $Q_{0}$ is an $\ell \times \ell$ matrix with $(1 \times \ell)$ rows $Q_{0, s}=\nabla^{\prime} \lambda\left(\bar{\alpha}_{(s)}\right)$, where $\bar{\alpha}_{(s)}$ is a mean value (different for each $s$ ) lying on the segment connecting $\alpha$ and $\alpha^{*}, s=1, \ldots, \ell$. It is straightforward to show that the correct specification ensures that $\lambda\left(\alpha^{*}\right)$ is zero. We will also show that

$$
Q_{0}=-Q^{*}+O\left(\left\|\alpha-\alpha^{*}\right\|\right),
$$

where $Q^{*}:=\sum_{i=1}^{n} \sum_{j=1}^{p} E\left[f_{i, j, t}(0) \nabla q_{i, j, t}\left(\cdot, \alpha^{*}\right) \nabla^{\prime} q_{i, j, t}\left(\cdot, \alpha^{*}\right)\right]$ with $f_{i, j, t}(0)$ representing the value at zero of the density $f_{i, j, t}$ of $\varepsilon_{i, j, t}:=Y_{i t}-q_{i, j, t}\left(\cdot, \alpha^{*}\right)$, conditional on $\mathcal{F}_{t-1}$. Combining equations (10) and (11) and putting $\lambda\left(\alpha^{*}\right)=$ 0 , we obtain

$$
\lambda(\alpha)=-Q^{*}\left(\alpha-\alpha^{*}\right)+O\left(\left\|\alpha-\alpha^{*}\right\|^{2}\right) .
$$

The next step is to show that

$$
T^{1 / 2} \lambda\left(\hat{\alpha}_{T}\right)+H_{T}=o_{p}(1),
$$

where $H_{T}:=T^{-1 / 2} \sum_{t=1}^{T} \eta_{t}^{*}$, with $\eta_{t}^{*}:=\sum_{i=1}^{n} \sum_{j=1}^{p} \nabla q_{i, j, t}\left(\cdot, \alpha^{*}\right) \psi_{\theta_{i j}}\left(\varepsilon_{i, j, t}\right)$. Equations (12) and (13) then yield the following asymptotic representation of our estimator $\hat{\alpha}_{T}$ :

$$
T^{1 / 2}\left(\hat{\alpha}_{T}-\alpha^{*}\right)=Q^{*-1} T^{-1 / 2} \sum_{t=1}^{T} \eta_{t}^{*}+o_{p}(1) .
$$

As we impose conditions sufficient to ensure that $\left\{\eta_{t}^{*}, \mathcal{F}_{t}\right\}$ is a martingale difference sequence (MDS), a suitable central limit theorem (e.g., theorem 5.24 in White, 2001) is applied to equation (14) to yield the desired asymptotic normality of $\hat{\alpha}_{T}$ :

$$
T^{1 / 2}\left(\hat{\alpha}_{T}-\alpha^{*}\right) \stackrel{d}{\rightarrow} N\left(0, Q^{*-1} V^{*} Q^{*-1}\right),
$$

where $V^{*}:=E\left(\eta_{t}^{*} \eta_{t}^{* \prime}\right)$.

We now strengthen the conditions given in the text to ensure that each step of the above argument is valid. 
Assumption 2 (iii) (a) There exists a finite positive constant $f_{0}$ such that for each $i$ and $t$, each $\omega \in \Omega$, and each $y \in \mathbb{R}, f_{i t}(\omega, y) \leq f_{0}<\infty$; (b) There exists a finite positive constant $L_{0}$ such that for each $i$ and $t$, each $\omega \in \Omega$, and each $y_{1}, y_{2} \in \mathbb{R},\left|f_{i t}\left(\omega, y_{1}\right)-f_{i t}\left(\omega, y_{2}\right)\right| \leq L_{0}\left|y_{1}-y_{2}\right|$.

Next we impose sufficient differentiability of $q_{t}$ with respect to $\alpha$.

Assumption 3 (ii) For each $t$ and each $\omega \in \Omega, q_{t}(\omega, \cdot)$ is continuously differentiable on $\mathbb{A}$; (iii) For each $t$ and each $\omega \in \Omega, q_{t}(\omega, \cdot)$ is twice continuously differentiable on $\mathbb{A}$.

To exploit the mean value theorem, we require that $\alpha^{*}$ belongs to $\operatorname{int}(\mathbb{A})$, the interior of $\mathbb{A}$.

Assumption 4 (ii) $\alpha^{*} \in \operatorname{int}(\mathbb{A})$.

Next, we place domination conditions on the derivatives of $q_{t}$.

Assumption 5 (iii) Let $D_{1, t}:=\max _{i=1, \ldots, n} \max _{j=1, \ldots, p} \max _{s=1, \ldots, \ell} \sup _{\alpha \in \mathbb{A}}\left|\left(\partial / \partial \alpha_{s}\right) q_{i, j, t}(\cdot, \alpha)\right|$. Then (a) $E\left(D_{1, t}\right)<\infty$; (b) $E\left(D_{1, t}^{2}\right)<\infty$; (iv) Let us define

$$
D_{2, t}:=\max _{i=1, \ldots, n} \max _{j=1, \ldots, p} \max _{s=1, \ldots, \ell} \max _{h=1, \ldots, \ell} \sup _{\alpha \in \mathbb{A}}\left|\left(\partial^{2} / \partial \alpha_{s} \partial \alpha_{h}\right) q_{i, j, t}(\cdot, \alpha)\right| .
$$

Then (a) $E\left(D_{2, t}\right)<\infty$; (b) $E\left(D_{2, t}^{2}\right)<\infty$.

Assumption 6 (i) $Q^{*}:=\sum_{i=1}^{n} \sum_{j=1}^{p} E\left[f_{i, j, t}(0) \nabla q_{i, j, t}\left(\cdot, \alpha^{*}\right) \nabla^{\prime} q_{i, j, t}\left(\cdot, \alpha^{*}\right)\right]$ is positive definite; (ii) $V^{*}:=E\left(\eta_{t}^{*} \eta_{t}^{* \prime}\right)$ is positive definite.

Assumptions 3(ii) and 5(iii.a) are additional assumptions that help to ensure that equation (9) holds. Further imposing Assumptions 2(iii), 3(iii.a), 4(ii), and 5(iv.a) suffices to ensure that equation (12) holds. The additional regularity provided by Assumptions 5(iii.b), 5(iv.b), and 6(i) ensures that equation (13) holds. Assumptions 5(iii.b) and 6(ii) help ensure the availability of the MDS central limit theorem. We now have conditions that are sufficient to prove the asymptotic normality of our MVMQ-CAViaR estimator.

Proof of Theorem 2 As outlined above, we first prove

$$
T^{-1} \sum_{t=1}^{T}\left\{\sum_{i=1}^{n} \sum_{j=1}^{p} \nabla q_{i, j, t}\left(\cdot, \hat{\alpha}_{T}\right) \psi_{\theta_{i j}}\left(Y_{i t}-q_{i, j, t}\left(\cdot, \hat{\alpha}_{T}\right)\right)\right\}=o_{p}(1) .
$$

The existence of $\nabla q_{i, j, t}$ is ensured by Assumption 3(ii). Let $e_{i}$ be the $\ell \times 1$ 
unit vector with $i^{\text {th }}$ element equal to one and the rest zero, and let

$$
G_{s}(c):=T^{-1 / 2} \sum_{t=1}^{T} \sum_{i=1}^{n} \sum_{j=1}^{p} \rho_{\theta_{i j}}\left(Y_{i t}-q_{i, j, t}\left(\cdot, \hat{\alpha}_{T}+c e_{s}\right)\right),
$$

for any real number $c$. Then, by the definition of $\hat{\alpha}_{T}, G_{s}(c)$ is minimized at $c=0$. Let $H_{s}(c)$ be the derivative of $G_{s}(c)$ with respect to $c$ from the right. Then

$H_{s}(c)=-T^{-1 / 2} \sum_{t=1}^{T} \sum_{i=1}^{n} \sum_{j=1}^{p} \nabla_{s} q_{i, j, t}\left(\cdot, \hat{\alpha}_{T}+c e_{s}\right) \psi_{\theta_{i j}}\left(Y_{i t}-q_{i, j, t}\left(\cdot, \hat{\alpha}_{T}+c e_{s}\right)\right)$,

where $\nabla_{s} q_{i, j, t}\left(\cdot, \hat{\alpha}_{T}+c e_{s}\right)$ is the $s^{t h}$ element of $\nabla q_{i, j, t}\left(\cdot, \hat{\alpha}_{T}+c e_{s}\right)$. Using the facts that (i) $H_{s}(c)$ is non-decreasing in $c$ and (ii) for any $\epsilon>0, H_{s}(-\epsilon) \leq 0$ and $H_{s}(\epsilon) \geq 0$, we have

$$
\begin{aligned}
\left|H_{s}(0)\right| & \leq H_{s}(\epsilon)-H_{s}(-\epsilon) \\
& \leq T^{-1 / 2} \sum_{t=1}^{T} \sum_{i=1}^{n} \sum_{j=1}^{p}\left|\nabla_{s} q_{i, j, t}\left(\cdot, \hat{\alpha}_{T}\right)\right| 1_{\left[Y_{i t}-q_{i, j, t}\left(\cdot, \hat{\alpha}_{T}\right)=0\right]} \\
& \leq T^{-1 / 2} \max _{1 \leq t \leq T} D_{1, t} \sum_{t=1}^{T} \sum_{i=1}^{n} \sum_{j=1}^{p} 1_{\left[Y_{i t}-q_{i, j, t}\left(\cdot, \hat{\alpha}_{T}\right)=0\right]},
\end{aligned}
$$

where the last inequality follows from the domination condition imposed in Assumption 5(iii.a). Because $D_{1, t}$ is stationary, $T^{-1 / 2} \max _{1 \leq t \leq T} D_{1, t}=$ $o_{p}(1)$. The second term is bounded in probability: $\sum_{t=1}^{T} \sum_{i=1}^{n} \sum_{j=1}^{p} 1_{\left[Y_{i t}-q_{i, j, t}\left(\cdot, \hat{\alpha}_{T}\right)=0\right]}=$ $O_{p}(1)$ given Assumption 2(i,ii.a) (see Koenker and Bassett, 1978, for details). Since $H_{s}(0)$ is the $s^{\text {th }}$ element of $T^{-1 / 2} \sum_{t=1}^{T} \sum_{i=1}^{n} \sum_{j=1}^{p} \nabla q_{i, j, t}\left(\cdot, \hat{\alpha}_{T}\right)$ $\psi_{\theta_{i j}}\left(Y_{i t}-q_{i, j, t}\left(\cdot, \hat{\alpha}_{T}\right)\right)$, the claim in (16) is proven.

Next, for each $\alpha \in \mathbb{A}$, Assumptions 3(ii) and 5(iii.a) ensure the existence and finiteness of the $\ell \times 1$ vector

$$
\begin{aligned}
\lambda(\alpha) & :=\sum_{i=1}^{n} \sum_{j=1}^{p} E\left[\nabla q_{i, j, t}(\cdot, \alpha) \psi_{\theta_{i j}}\left(Y_{i t}-q_{i, j, t}(\cdot, \alpha)\right)\right] \\
& =\sum_{i=1}^{n} \sum_{j=1}^{p} E\left[\nabla q_{i, j, t}(\cdot, \alpha) \int_{\delta_{i, j, t}\left(\alpha, \alpha^{*}\right)}^{0} f_{i, j, t}(s) d s\right],
\end{aligned}
$$

where $\delta_{i, j, t}\left(\alpha, \alpha^{*}\right):=q_{i, j, t}(\cdot, \alpha)-q_{i, j, t}\left(\cdot, \alpha^{*}\right)$ and $f_{i, j, t}(s)=(d / d s) F_{i t}(s+$ $\left.q_{i, j, t}\left(\cdot, \alpha^{*}\right)\right)$ represents the conditional density of $\varepsilon_{i, j, t}:=Y_{i t}-q_{i, j, t}\left(\cdot, \alpha^{*}\right)$ with respect to Lebesgue measure. The differentiability and domination conditions provided by Assumptions 3(iii) and 5(iv.a) ensure (e.g., by Bartle, 1966, corollary 5.9) the continuous differentiability of $\lambda(\alpha)$ on $\mathbb{A}$, with

$$
\nabla \lambda(\alpha)=\sum_{i=1}^{n} \sum_{j=1}^{p} E\left[\nabla\left\{\nabla^{\prime} q_{i, j, t}(\cdot, \alpha) \int_{\delta_{i, j, t}\left(\alpha, \alpha^{*}\right)}^{0} f_{i, j, t}(s) d s\right\}\right] .
$$


Since $\alpha^{*}$ is interior to $\mathbb{A}$ by Assumption 4(ii), the mean value theorem applies to each element of $\lambda(\alpha)$ to yield

$$
\lambda(\alpha)=\lambda\left(\alpha^{*}\right)+Q_{0}\left(\alpha-\alpha^{*}\right),
$$

for $\alpha$ in a convex compact neighborhood of $\alpha^{*}$, where $Q_{0}$ is an $\ell \times \ell$ matrix with $(1 \times \ell)$ rows $Q_{s}\left(\bar{\alpha}_{(s)}\right)=\nabla^{\prime} \lambda\left(\bar{\alpha}_{(s)}\right)$, where $\bar{\alpha}_{(s)}$ is a mean value (different for each $s$ ) lying on the segment connecting $\alpha$ and $\alpha^{*}$ with $s=1, \ldots, \ell$. The chain rule and an application of the Leibniz rule to $\int_{\delta_{i, j, t}\left(\alpha, \alpha^{*}\right)}^{0} f_{i, j, t}(s) d s$ then give

$$
Q_{s}(\alpha)=A_{s}(\alpha)-B_{s}(\alpha)
$$

where

$$
\begin{aligned}
A_{s}(\alpha) & :=\sum_{i=1}^{n} \sum_{j=1}^{p} E\left[\nabla_{s} \nabla^{\prime} q_{i, j, t}(\cdot, \alpha) \int_{\delta_{i, j, t}\left(\alpha, \alpha^{*}\right)}^{0} f_{i, j, t}(s) d s\right] \\
B_{s}(\alpha) & :=\sum_{i=1}^{n} \sum_{j=1}^{p} E\left[f_{i, j, t}\left(\delta_{i, j, t}\left(\alpha, \alpha^{*}\right)\right) \nabla_{s} q_{i, j, t}(\cdot, \alpha) \nabla^{\prime} q_{i, j, t}(\cdot, \alpha)\right] .
\end{aligned}
$$

Assumption 2(iii) and the other domination conditions (those of Assumption 5) then ensure that

$$
\begin{aligned}
& A_{s}\left(\bar{\alpha}_{(s)}\right)=O\left(\left\|\alpha-\alpha^{*}\right\|\right) \\
& B_{s}\left(\bar{\alpha}_{(s)}\right)=Q_{s}^{*}+O\left(\left\|\alpha-\alpha^{*}\right\|\right),
\end{aligned}
$$

where $Q_{s}^{*}:=\sum_{i=1}^{n} \sum_{j=1}^{p} E\left[f_{i, j, t}(0) \nabla_{s} q_{i, j, t}\left(\cdot, \alpha^{*}\right) \nabla^{\prime} q_{i, j, t}\left(\cdot, \alpha^{*}\right)\right]$. Letting $Q^{*}:=$ $\sum_{i=1}^{n} \sum_{j=1}^{p}$

$E\left[f_{i, j, t}(0) \nabla q_{i, j, t}\left(\cdot, \alpha^{*}\right) \nabla^{\prime} q_{i, j, t}\left(\cdot, \alpha^{*}\right)\right]$, we obtain

$$
Q_{0}=-Q^{*}+O\left(\left\|\alpha-\alpha^{*}\right\|\right) .
$$

Next, we have that $\lambda\left(\alpha^{*}\right)=0$. To show this, we write

$$
\begin{aligned}
\lambda\left(\alpha^{*}\right) & =\sum_{i=1}^{n} \sum_{j=1}^{p} E\left[\nabla q_{i, j, t}\left(\cdot, \alpha^{*}\right) \psi_{\theta_{i j}}\left(Y_{i t}-q_{i, j, t}\left(\cdot, \alpha^{*}\right)\right)\right] \\
& =\sum_{i=1}^{n} \sum_{j=1}^{p} E\left(E\left[\nabla q_{i, j, t}\left(\cdot, \alpha^{*}\right) \psi_{\theta_{i j}}\left(Y_{i t}-q_{i, j, t}\left(\cdot, \alpha^{*}\right)\right) \mid \mathcal{F}_{t-1}\right]\right) \\
& =\sum_{i=1}^{n} \sum_{j=1}^{p} E\left(\nabla q_{i, j, t}\left(\cdot, \alpha^{*}\right) E\left[\psi_{\theta_{i j}}\left(Y_{i t}-q_{i, j, t}\left(\cdot, \alpha^{*}\right)\right) \mid \mathcal{F}_{t-1}\right]\right) \\
& =\sum_{i=1}^{n} \sum_{j=1}^{p} E\left(\nabla q_{i, j, t}\left(\cdot, \alpha^{*}\right) E\left[\psi_{\theta_{i j}}\left(\varepsilon_{i, j, t}\right) \mid \mathcal{F}_{t-1}\right]\right) \\
& =0
\end{aligned}
$$


as $E\left[\psi_{\theta_{i j}}\left(\varepsilon_{i, j, t}\right) \mid \mathcal{F}_{t-1}\right]=\theta_{i j}-E\left[1_{\left[Y_{i t} \leq q_{i, j, t}^{*}\right]} \mid \mathcal{F}_{t-1}\right]=0$, by definition of $q_{i, j, t}^{*}$ for $i=1, \ldots, n$ and $j=1, \ldots, p$ (see equation (3)). Combining $\lambda\left(\alpha^{*}\right)=0$ with equations (17) and (18), we obtain

$$
\lambda(\alpha)=-Q^{*}\left(\alpha-\alpha^{*}\right)+O\left(\left\|\alpha-\alpha^{*}\right\|^{2}\right) .
$$

The next step is to show that

$$
T^{1 / 2} \lambda\left(\hat{\alpha}_{T}\right)+H_{T}=o_{p}(1)
$$

where $H_{T}:=T^{-1 / 2} \sum_{t=1}^{T} \eta_{t}^{*}$, with $\eta_{t}^{*}:=\eta_{t}\left(\alpha^{*}\right)$ and $\eta_{t}(\alpha):=\sum_{i=1}^{n} \sum_{j=1}^{p} \nabla q_{i, j, t}(\cdot, \alpha)$ $\psi_{\theta_{i j}}\left(Y_{i t}-q_{i, j, t}(\cdot, \alpha)\right)$. Let $u_{t}(\alpha, d):=\sup _{\{\tau:\|\tau-\alpha\| \leq d\}}\left\|\eta_{t}(\tau)-\eta_{t}(\alpha)\right\|$. By the results of Huber (1967) and Weiss (1991), to prove (20) it suffices to show the following: (i) there exist $a>0$ and $d_{0}>0$ such that $\|\lambda(\alpha)\| \geq a\left\|\alpha-\alpha^{*}\right\|$ for $\left\|\alpha-\alpha^{*}\right\| \leq d_{0}$; (ii) there exist $b>0, d_{0}>0$, and $d \geq 0$ such that $E\left[u_{t}(\alpha, d)\right] \leq b d$ for $\left\|\alpha-\alpha^{*}\right\|+d \leq d_{0}$; and (iii) there exist $c>0, d_{0}>0$, and $d \geq 0$ such that $E\left[u_{t}(\alpha, d)^{2}\right] \leq c d$ for $\left\|\alpha-\alpha^{*}\right\|+d \leq d_{0}$.

The condition that $Q^{*}$ is positive-definite in Assumption 6(i) is sufficient for (i). For (ii), we have that for the given (small) $d>0$

$$
\begin{aligned}
& u_{t}(\alpha, d) \\
& \leq \sup _{\{\tau:\|\tau-\alpha\| \leq d\}} \sum_{i=1}^{n} \sum_{j=1}^{p}\left\|\nabla q_{i, j, t}(\cdot, \tau) \psi_{\theta_{i j}}\left(Y_{i t}-q_{i, j, t}(\cdot, \tau)\right)-\nabla q_{i, j, t}(\cdot, \alpha) \psi_{\theta_{i j}}\left(Y_{i t}-q_{i, j, t}(\cdot, \alpha)\right)\right\| \\
& \leq \sum_{i=1}^{n} \sum_{j=1}^{p} \sup _{\{\tau:\|\tau-\alpha\| \leq d\}}\left\|\psi_{\theta_{i j}}\left(Y_{i t}-q_{i, j, t}(\cdot, \tau)\right)\right\| \times \sup _{\{\tau:\|\tau-\alpha\| \leq d\}}\left\|\nabla q_{i, j, t}(\cdot, \tau)-\nabla q_{i, j, t}(\cdot, \alpha)\right\| \\
& \quad+\sum_{i=1}^{n} \sum_{j=1}^{p} \sup _{\{\tau:\|\tau-\alpha\| \leq d\}}\left\|\psi_{\theta_{i j}}\left(Y_{i t}-q_{i, j, t}(\cdot, \alpha)\right)-\psi_{\theta_{i j}}\left(Y_{i t}-q_{i, j, t}(\cdot, \tau)\right)\right\| \\
& \quad \times \sup _{\{\tau:\|\tau-\alpha\| \leq d\}}\left\|\nabla q_{i, j, t}(\cdot, \alpha)\right\| \\
& \leq n p D_{2, t} d+D_{1, t} \sum_{i=1}^{n} \sum_{j=1}^{p} 1_{\left[\left|Y_{i t}-q_{i, j, t}(\cdot, \alpha)\right|<D_{1, t} d\right]}
\end{aligned}
$$

using the following: (i) $\left\|\psi_{\theta_{i j}}\left(Y_{i t}-q_{i, j, t}(\cdot, \tau)\right)\right\| \leq 1$; (ii) $\| \psi_{\theta_{i j}}\left(Y_{i t}-q_{i, j, t}(\cdot, \alpha)\right)-$ $\psi_{\theta_{i j}}\left(Y_{i t}-q_{i, j, t}(\cdot, \tau)\right)|| \leq 1_{\left[\left|Y_{i t}-q_{i, j, t}(\cdot, \alpha)\right|<\left|q_{i, j, t}(\cdot, \tau)-q_{i, j, t}(\cdot, \alpha)\right|\right]}$; and (iii) the mean value theorem applied to $\nabla q_{i, j, t}(\cdot, \tau)$ and $q_{i, j, t}(\cdot, \alpha)$. Hence, we have

$$
E\left[u_{t}(\alpha, d)\right] \leq n p C_{0} d+2 n p C_{1} f_{0} d
$$

for some constants $C_{0}$ and $C_{1}$, given Assumptions 2(iii.a), 5(iii.a), and 5 (iv.a). Hence, (ii) holds for $b=n p C_{0}+2 n p C_{1} f_{0}$ and $d_{0}=2 d$. The last condition (iii) can be similarly verified by applying the $c_{r}$-inequality to equation (??) with $d<1$ (so that $d^{2}<d$ ) and using Assumptions 2(iii.a), 5 (iii.b), and 5(iv.b). As a result, equation (20) is verified. 
Combining equations (19) and (20) yields

$$
Q^{*} T^{1 / 2}\left(\hat{\alpha}_{T}-\alpha^{*}\right)=T^{-1 / 2} \sum_{t=1}^{T} \eta_{t}^{*}+o_{p}(1) .
$$

However, $\left\{\eta_{t}^{*}, \mathcal{F}_{t}\right\}$ is a stationary ergodic martingale difference sequence (MDS). In particular, $\eta_{t}^{*}$ is measurable $-\mathcal{F}_{t}$, and $E\left(\eta_{t}^{*} \mid \mathcal{F}_{t-1}\right)=E\left(\sum_{i=1}^{n} \sum_{j=1}^{p} \nabla q_{i, j, t}\left(\cdot, \alpha^{*}\right) \psi_{\theta_{i j}}\left(\varepsilon_{i, j, t}\right)\right.$ $\left.\mid \mathcal{F}_{t-1}\right)=\sum_{i=1}^{n} \sum_{j=1}^{p} \nabla q_{i, j, t}\left(\cdot, \alpha^{*}\right) E\left(\psi_{\theta_{i j}}\left(\varepsilon_{i, j, t}\right) \mid \mathcal{F}_{t-1}\right)=0$, as $E\left[\psi_{\theta_{i j}}\left(\varepsilon_{i, j, t}\right) \mid\right.$ $\left.\mathcal{F}_{t-1}\right]=0$ for all $i=1, \ldots, n$ and $j=1, \ldots, p$. Assumption 5(iii.b) ensures that $V^{*}:=E\left(\eta_{t}^{*} \eta_{t}^{* \prime}\right)$ is finite. The MDS central limit theorem (e.g., theorem 5.24 of White, 2001) applies, provided $V^{*}$ is positive definite (as ensured by Assumption 6(ii)) and that $T^{-1} \sum_{t=1}^{T} \eta_{t}^{*} \eta_{t}^{* \prime}=V^{*}+o_{p}(1)$, which is ensured by the ergodic theorem. The standard argument now gives

$$
V^{*-1 / 2} Q^{*} T^{1 / 2}\left(\hat{\alpha}_{T}-\alpha^{*}\right) \stackrel{d}{\rightarrow} N(0, I),
$$

which completes the proof.

Proof of Theorem 3 We have

$$
\hat{V}_{T}-V^{*}=\left(T^{-1} \sum_{t=1}^{T} \hat{\eta}_{t} \hat{\eta}_{t}^{\prime}-T^{-1} \sum_{t=1}^{T} \eta_{t}^{*} \eta_{t}^{* \prime}\right)+\left(T^{-1} \sum_{t=1}^{T} \eta_{t}^{*} \eta_{t}^{* \prime}-E\left[\eta_{t}^{*} \eta_{t}^{* \prime}\right]\right),
$$

where $\hat{\eta}_{t}:=\sum_{i=1}^{n} \sum_{j=1}^{p} \nabla \hat{q}_{i, j, t} \hat{\psi}_{i, j, t}$ and $\eta_{t}^{*}:=\sum_{i=1}^{n} \sum_{j=1}^{p} \nabla q_{i, j, t}^{*} \psi_{i, j, t}^{*}$, with $\nabla \hat{q}_{i, j, t}:=\nabla q_{i, j, t}\left(\cdot, \hat{\alpha}_{T}\right), \hat{\psi}_{i, j, t}:=\psi_{\theta_{i j}}\left(Y_{i t}-q_{i, j, t}\left(\cdot, \hat{\alpha}_{T}\right)\right), \nabla q_{i, j, t}^{*}:=\nabla q_{i, j, t}\left(\cdot, \alpha^{*}\right)$, and $\psi_{i, j, t}^{*}:=\psi_{\theta_{i j}}\left(Y_{i t}-q_{i, j, t}\left(\cdot, \alpha^{*}\right)\right)$. Assumptions 1 and $2(\mathrm{i}, \mathrm{ii})$ ensure that $\left\{\eta_{t}^{*} \eta_{t}^{* \prime}\right\}$ is a stationary ergodic sequence. Assumptions 3(i,ii), 4(i.a), and 5 (iii) ensure that $E\left[\eta_{t}^{*} \eta_{t}^{* \prime}\right]<\infty$. It follows by the ergodic theorem that $T^{-1} \sum_{t=1}^{T} \eta_{t}^{*} \eta_{t}^{* \prime}-E\left[\eta_{t}^{*} \eta_{t}^{* \prime}\right]=o_{p}(1)$. Thus, it suffices to prove $T^{-1} \sum_{t=1}^{T} \hat{\eta}_{t} \hat{\eta}_{t}^{\prime}-$ $T^{-1} \sum_{t=1}^{T} \eta_{t}^{*} \eta_{t}^{* \prime}=o_{p}(1)$.

The $(h, s)$ element of $T^{-1} \sum_{t=1}^{T} \hat{\eta}_{t} \hat{\eta}_{t}^{\prime}-T^{-1} \sum_{t=1}^{T} \eta_{t}^{*} \eta_{t}^{* \prime}$ is $T^{-1} \sum_{t=1}^{T}\left\{\sum_{i=1}^{n} \sum_{j=1}^{p} \sum_{l=1}^{n} \sum_{k=1}^{p}\left(\hat{\psi}_{i, j, t} \hat{\psi}_{l, k, t} \nabla_{h} \hat{q}_{i, j, t} \nabla_{s} \hat{q}_{l, k, t}-\psi_{i, j, t}^{*} \psi_{l, k, t}^{*} \nabla_{h} q_{i, j, t}^{*} \nabla_{s} q_{l, k, t}^{*}\right)\right\}$.

Thus, it will suffice to show that for each $(h, s)$ and $(i, j, l, k)$,

$$
T^{-1} \sum_{t=1}^{T}\left\{\hat{\psi}_{i, j, t} \hat{\psi}_{l, k, t} \nabla_{h} \hat{q}_{i, j, t} \nabla_{s} \hat{q}_{l, k, t}-\psi_{i, j, t}^{*} \psi_{l, k, t}^{*} \nabla_{h} q_{i, j, t}^{*} \nabla_{s} q_{l, k, t}^{*}\right\}=o_{p}(1) .
$$

By the triangle inequality,

$$
\left|T^{-1} \sum_{t=1}^{T}\left\{\hat{\psi}_{i, j, t} \hat{\psi}_{l, k, t} \nabla_{h} \hat{q}_{i, j, t} \nabla_{s} \hat{q}_{l, k, t}-\psi_{i, j, t}^{*} \psi_{l, k, t}^{*} \nabla_{h} q_{i, j, t}^{*} \nabla_{s} q_{l, k, t}^{*}\right\}\right| \leq A_{T}+B_{T},
$$


where

$$
\begin{aligned}
& A_{T}=T^{-1} \sum_{t=1}^{T}\left|\hat{\psi}_{i, j, t} \hat{\psi}_{l, k, t} \nabla_{h} \hat{q}_{i, j, t} \nabla_{s} \hat{q}_{l, k, t}-\psi_{i, j, t}^{*} \psi_{l, k, t}^{*} \nabla_{h} \hat{q}_{i, j, t} \nabla_{s} \hat{q}_{l, k, t}\right| \\
& B_{T}=T^{-1} \sum_{t=1}^{T}\left|\psi_{i, j, t}^{*} \psi_{l, k, t}^{*} \nabla_{h} q_{i, j, t}^{*} \nabla_{s} q_{l, k, t}^{*}-\psi_{i, j, t}^{*} \psi_{l, k, t}^{*} \nabla_{h} \hat{q}_{i, j, t} \nabla_{s} \hat{q}_{l, k, t}\right| .
\end{aligned}
$$

We now show that $A_{T}=o_{p}(1)$ and $B_{T}=o_{p}(1)$, delivering the desired result. For $A_{T}$, the triangle inequality gives

$$
A_{T} \leq A_{1 T}+A_{2 T}+A_{3 T}
$$

where

$$
\begin{aligned}
& A_{1 T}=T^{-1} \sum_{t=1}^{T} \theta_{i j}\left|1_{\left[\varepsilon_{i, j, t} \leq 0\right]}-1_{\left[\hat{\varepsilon}_{i, j, t} \leq 0\right]}\right|\left|\nabla_{h} \hat{q}_{i, j, t} \nabla_{s} \hat{q}_{l, k, t}\right| \\
& A_{2 T}=T^{-1} \sum_{t=1}^{T} \theta_{l k}\left|1_{\left[\varepsilon_{l, k, t} \leq 0\right]}-1_{\left[\hat{\varepsilon}_{l, k, t} \leq 0\right]}\right|\left|\nabla_{h} \hat{q}_{i, j, t} \nabla_{s} \hat{q}_{l, k, t}\right| \\
& A_{3 T}=T^{-1} \sum_{t=1}^{T}\left|1_{\left[\varepsilon_{i, j, t} \leq 0\right]} 1_{\left[\varepsilon_{l, k, t} \leq 0\right]}-1_{\left[\hat{\varepsilon}_{i, j, t} \leq 0\right]} 1_{\left[\hat{\varepsilon}_{l, k, t} \leq 0\right]}\right|\left|\nabla_{h} \hat{q}_{i, j, t} \nabla_{s} \hat{q}_{l, k, t}\right| .
\end{aligned}
$$

Theorem 2, ensured by Assumptions $1-6$, implies that $T^{1 / 2}\left\|\hat{\alpha}_{T}-\alpha^{*}\right\|=$ $O_{p}(1)$. This, together with Assumptions 2(iii,iv) and 5(iii.b), enables us to apply the same techniques used in Kim and White (2003) to show $A_{1 T}=$ $o_{p}(1), A_{2 T}=o_{p}(1)$, and $A_{3 T}=o_{p}(1)$, implying $A_{T}=o_{p}(1)$.

It remains to show $B_{T}=o_{p}(1)$. By the triangle inequality,

$$
B_{T} \leq B_{1 T}+B_{2 T}
$$

where

$$
\begin{aligned}
& B_{1 T}=T^{-1} \sum_{t=1}^{T}\left|\psi_{i, j, t}^{*} \psi_{l, k, t}^{*} \nabla_{h} q_{i, j, t}^{*} \nabla_{s} q_{l, k, t}^{*}-E\left[\psi_{i, j, t}^{*} \psi_{l, k, t}^{*} \nabla_{h} q_{i, j, t}^{*} \nabla_{s} q_{l, k, t}^{*}\right]\right| \\
& B_{2 T}=T^{-1} \sum_{t=1}^{T}\left|\psi_{i, j, t}^{*} \psi_{l, k, t}^{*} \nabla_{h} \hat{q}_{i, j, t} \nabla_{s} \hat{q}_{l, k, t}-E\left[\psi_{i, j, t}^{*} \psi_{l, k, t}^{*} \nabla_{h} q_{i, j, t}^{*} \nabla_{s} q_{l, k, t}^{*}\right]\right| .
\end{aligned}
$$

Assumptions 1, 2(i,ii), 3(i,ii), 4(i.a), and 5(iii) ensure that the ergodic theorem applies to the stochastic sequence of $\left\{\psi_{i, j, t}^{*} \psi_{l, k, t}^{*} \nabla_{h} q_{i, j, t}^{*} \nabla_{s} q_{l, k, t}^{*}\right\}$, so $B_{1 T}=o_{p}(1)$. Next, Assumptions 1, 3(i,ii), and 5(iii) ensure that the stationary ergodic ULLN applies to $\left\{\psi_{i, j, t}^{*} \psi_{l, k, t}^{*} \nabla_{h} q_{i, j, t}(\cdot, \alpha)\right.$

$\left.\nabla_{s} q_{l, k, t}(\cdot, \alpha)\right\}$. This and the result of Theorem $1\left(\hat{\alpha}_{T}-\alpha^{*}=o_{p}(1)\right)$ ensure that $B_{2 T}=o_{p}(1)$ by e.g., White (1994, corollary 3.8), and the proof is complete. 
To establish the consistency of $\hat{Q}_{T}$, we strengthen the domination condition on $\nabla q_{i, j, t}$ and impose conditions on $\left\{\hat{c}_{T}\right\}$.

Assumption 5 (iii) (c) $E\left(D_{1, t}^{3}\right)<\infty$.

Assumption $7\left\{\hat{c}_{T}\right\}$ is a stochastic sequence and $\left\{c_{T}\right\}$ is a non-stochastic sequence such that (i) $\hat{c}_{T} / c_{T} \stackrel{p}{\rightarrow} 1$; (ii) $c_{T}=o(1)$; and (iii) $c_{T}^{-1}=o\left(T^{1 / 2}\right)$.

Proof of Theorem 4 We begin by sketching the proof. We first define

$$
Q_{T}:=\left(2 c_{T} T\right)^{-1} \sum_{t=1}^{T} \sum_{i=1}^{n} \sum_{j=1}^{p} 1_{\left[-c_{T} \leq \varepsilon_{i, j, t} \leq c_{T}\right]} \nabla q_{i, j, t}^{*} \nabla^{\prime} q_{i, j, t}^{*},
$$

and then we will show the following:

$$
\begin{aligned}
& Q^{*}-E\left(Q_{T}\right) \stackrel{p}{\rightarrow} 0, \\
& E\left(Q_{T}\right)-Q_{T} \stackrel{p}{\rightarrow} 0, \\
& Q_{T}-\hat{Q}_{T} \stackrel{p}{\rightarrow} 0 .
\end{aligned}
$$

Combining the results above will deliver the desired outcome: $\hat{Q}_{T}-Q^{*} \stackrel{p}{\rightarrow} 0$.

For $(21)$, one can show by applying the mean value theorem to $F_{i, j, t}\left(c_{T}\right)-$ $F_{i, j, t}\left(-c_{T}\right)$, where $F_{i, j, t}(c):=\int 1_{\{s \leq c\}} f_{i, j, t}(s) d s$, that

$E\left(Q_{T}\right)=T^{-1} \sum_{t=1}^{T} \sum_{i=1}^{n} \sum_{j=1}^{p} E\left[f_{i, j, t}\left(\xi_{i, j, T}\right) \nabla q_{i, j, t}^{*} \nabla^{\prime} q_{i, j, t}^{*}\right]=\sum_{i=1}^{n} \sum_{j=1}^{p} E\left[f_{i, j, t}\left(\xi_{i, j, T}\right) \nabla q_{i, j, t}^{*} \nabla^{\prime} q_{i, j, t}^{*}\right]$,

where $\xi_{i, j, T}$ is a mean value lying between $-c_{T}$ and $c_{T}$, and the second equality follows by stationarity. Therefore, the $(h, s)$ element of $\left|E\left(Q_{T}\right)-Q^{*}\right|$ satisfies

$$
\begin{aligned}
& \left|\sum_{i=1}^{n} \sum_{j=1}^{p} E\left\{f_{i, j, t}\left(\xi_{i, j, T}\right)-f_{i, j, t}(0) \nabla_{h} q_{i, j, t}^{*} \nabla_{s} q_{i, j, t}^{*}\right\}\right| \\
\leq & \sum_{i=1}^{n} \sum_{j=1}^{p} E\left\{\left|f_{i, j, t}\left(\xi_{i, j, T}\right)-f_{i, j, t}(0)\right|\left|\nabla_{h} q_{i, j, t}^{*} \nabla_{s} q_{i, j, t}^{*}\right|\right\} \\
\leq & \sum_{i=1}^{n} \sum_{j=1}^{p} L_{0} E\left\{\left|\xi_{i, j, T}\right|\left|\nabla_{h} q_{i, j, t}^{*} \nabla_{s} q_{i, j, t}^{*}\right|\right\} \\
\leq & n p L_{0} c_{T} E\left[D_{1, t}^{2}\right],
\end{aligned}
$$

which converges to zero as $c_{T} \rightarrow 0$. The second inequality follows from Assumption 2(iii.b), and the last inequality follows by Assumption 5(iii.b). Therefore, we have the result shown in equation (21). 
To show (22), it suffices to simply apply an LLN for double arrays, e.g. theorem 2 in Andrews (1988). Finally, for (23), we consider the $(h, s)$ element of $\left|\hat{Q}_{T}-Q_{T}\right|$, given by

$$
\begin{aligned}
& \mid \frac{1}{2 \hat{c}_{T} T} \sum_{t=1}^{T} \sum_{i=1}^{n} \sum_{j=1}^{p} 1_{\left[-\hat{c}_{T} \leq \hat{\varepsilon}_{i, j, t} \leq \hat{c}_{T}\right]} \nabla_{h} \hat{q}_{i, j, t} \nabla_{s} \hat{q}_{i, j, t} \\
& \quad-\frac{1}{2 c_{T} T} \sum_{t=1}^{T} \sum_{i=1}^{n} \sum_{j=1}^{p} 1_{\left[-c_{T} \leq \varepsilon_{i, j, t} \leq c_{T}\right]} \nabla_{h} q_{i, j, t}^{*} \nabla_{s} q_{i, j, t}^{*} \mid \\
& =\frac{c_{T}}{\hat{c}_{T}} \times \mid \frac{1}{2 c_{T} T} \sum_{t=1}^{T} \sum_{i=1}^{n} \sum_{j=1}^{p}\left(1_{\left[-\hat{c}_{T} \leq \hat{\varepsilon}_{i, j, t} \leq \hat{c}_{T}\right]}-1_{\left[-c_{T} \leq \varepsilon_{i, j, t} \leq c_{T}\right]}\right) \nabla_{h} \hat{q}_{i, j, t} \nabla_{s} \hat{q}_{i, j, t} \\
& \quad+\frac{1}{2 c_{T} T} \sum_{t=1}^{T} \sum_{i=1}^{n} \sum_{j=1}^{p} 1_{\left[-c_{T} \leq \varepsilon_{i, j, t} \leq c_{T}\right]}\left(\nabla_{h} \hat{q}_{i, j, t}-\nabla_{h} q_{i, j, t}^{*}\right) \nabla_{s} \hat{q}_{i, j, t} \\
& \quad+\frac{1}{2 c_{T} T} \sum_{t=1}^{T} \sum_{i=1}^{n} \sum_{j=1}^{p} 1_{\left[-c_{T} \leq \varepsilon_{i, j, t} \leq c_{T}\right]} \nabla_{h} q_{i, j, t}^{*}\left(\nabla_{s} \hat{q}_{i, j, t}-\nabla_{s} q_{i, j, t}^{*}\right) \\
& \quad+\frac{1}{2 c_{T} T}\left(1-\frac{\hat{c}_{T}}{c_{T}}\right) \sum_{t=1}^{T} \sum_{i=1}^{n} \sum_{j=1}^{p} 1_{\left[-c_{T} \leq \varepsilon_{i, j, t} \leq c_{T}\right]} \nabla_{h} q_{i, j, t}^{*} \nabla_{s} q_{i, j, t}^{*} \mid \\
& \leq \frac{c_{T}}{\hat{c}_{T}}\left[A_{1 T}+A_{2 T}+A_{3 T}+\left(1-\frac{\hat{c}_{T}}{c_{T}}\right) A_{4 T}\right]
\end{aligned}
$$

where

$$
\begin{aligned}
& A_{1 T}:=\frac{1}{2 c_{T} T} \sum_{t=1}^{T} \sum_{i=1}^{n} \sum_{j=1}^{p}\left|1_{\left[-\hat{c}_{T} \leq \hat{\varepsilon}_{i, j, t} \leq \hat{c}_{T}\right]}-1_{\left[-c_{T} \leq \varepsilon_{i, j, t} \leq c_{T}\right]}\right| \times\left|\nabla_{h} \hat{q}_{i, j, t} \nabla_{s} \hat{q}_{i, j, t}\right| \\
& A_{2 T}:=\frac{1}{2 c_{T} T} \sum_{t=1}^{T} \sum_{i=1}^{n} \sum_{j=1}^{p} 1_{\left[-c_{T} \leq \varepsilon_{i, j, t} \leq c_{T}\right]}\left|\nabla_{h} \hat{q}_{i, j, t}-\nabla_{h} q_{i, j, t}^{*}\right| \times\left|\nabla_{s} \hat{q}_{i, j, t}\right| \\
& A_{3 T}:=\frac{1}{2 c_{T} T} \sum_{t=1}^{T} \sum_{i=1}^{n} \sum_{j=1}^{p} 1_{\left[-c_{T} \leq \varepsilon_{i, j, t} \leq c_{T}\right]}\left|\nabla_{h} q_{i, j, t}^{*}\right| \times\left|\nabla_{s} \hat{q}_{i, j, t}-\nabla_{s} q_{i, j, t}^{*}\right| \\
& A_{4 T}:=\frac{1}{2 c_{T} T} \sum_{t=1}^{T} \sum_{i=1}^{n} \sum_{j=1}^{p} 1_{\left[-c_{T} \leq \varepsilon_{i, j, t} \leq c_{T}\right]}\left|\nabla_{h} q_{i, j, t}^{*} \nabla_{s} q_{i, j, t}^{*}\right| .
\end{aligned}
$$

It will suffice to show that $A_{1 T}=o_{p}(1), A_{2 T}=o_{p}(1), A_{3 T}=o_{p}(1)$, and $A_{4 T}=O_{p}(1)$. Then, because $\hat{c}_{T} / c_{T} \stackrel{p}{\rightarrow} 1$, we obtain the desired result: $\hat{Q}_{T}-Q^{*} \stackrel{p}{\rightarrow} 0$.

We first show $A_{1 T}=o_{p}(1)$. It will suffice to show that for each $i$ and $j$,

$$
\frac{1}{2 c_{T} T} \sum_{t=1}^{T}\left|1_{\left[-\hat{c}_{T} \leq \hat{\varepsilon}_{i, j, t} \leq \hat{c}_{T}\right]}-1_{\left[-c_{T} \leq \varepsilon_{i, j, t} \leq c_{T}\right]}\right| \times\left|\nabla_{h} \hat{q}_{i, j, t} \nabla_{s} \hat{q}_{i, j, t}\right|=o_{p}(1) .
$$


Let $\alpha_{T}$ lie between $\hat{\alpha}_{T}$ and $\alpha^{*}$, and put $d_{i, j, t, T}:=\left\|\nabla q_{i, j, t}\left(\cdot, \alpha_{T}\right)\right\| \times \| \hat{\alpha}_{T}-$ $\alpha^{*}||+\left|\hat{c}_{T}-c_{T}\right|$. Then

$\left(2 c_{T} T\right)^{-1} \sum_{t=1}^{T}\left|1_{\left[-\hat{c}_{T} \leq \hat{\varepsilon}_{i, j, t} \leq \hat{c}_{T}\right]}-1_{\left[-c_{T} \leq \varepsilon_{i, j, t} \leq c_{T}\right]}\right| \times\left|\nabla_{h} \hat{q}_{i, j, t} \nabla_{s} \hat{q}_{i, j, t}\right| \leq U_{T}+V_{T}$,

where

$$
\begin{aligned}
U_{T} & :=\left(2 c_{T} T\right)^{-1} \sum_{t=1}^{T} 1_{\left[\left|\varepsilon_{i, j, t}-c_{T}\right|<d_{i, j, t, T}\right]}\left|\nabla_{h} \hat{q}_{i, j, t} \nabla_{s} \hat{q}_{i, j, t}\right| \\
V_{T} & :=\left(2 c_{T} T\right)^{-1} \sum_{t=1}^{T} 1_{\left[\left|\varepsilon_{i, j, t}+c_{T}\right|<d_{i, j, t, T}\right]}\left|\nabla_{h} \hat{q}_{i, j, t} \nabla_{s} \hat{q}_{i, j, t}\right| .
\end{aligned}
$$

It will suffice to show that $U_{T} \stackrel{p}{\rightarrow} 0$ and $V_{T} \stackrel{p}{\rightarrow} 0$. Let $\eta>0$ and let $z$ be an arbitrary positive number. Then, using reasoning similar to that of Kim and White (2003, lemma 5), one can show that for any $\eta>0$,

$$
\begin{aligned}
P\left(U_{T}\right. & \left.>\eta) \leq P\left(\left(2 c_{T} T\right)^{-1} \sum_{t=1}^{T} 1_{\left[\left|\varepsilon_{i, j, t}-c_{T}\right|<\left(|| \nabla q_{i, j, t}\left(\cdot, \alpha_{T}\right) \|+1\right) z c_{T}\right]}\right)\left|\nabla_{h} \hat{q}_{i, j, t} \nabla_{s} \hat{q}_{i, j, t}\right|>\eta\right) \\
& \leq \frac{z f_{0}}{\eta T} \sum_{t=1}^{T} E\left\{\left(|| \nabla q_{i, j, t}\left(\cdot, \alpha_{T}\right) \|+1\right)\left|\nabla_{h} \hat{q}_{j, t} \nabla_{s} \hat{q}_{j, t}\right|\right\} \\
& \leq z f_{0}\left\{E\left|D_{1, t}^{3}\right|+E\left|D_{1, t}^{2}\right|\right\} / \eta,
\end{aligned}
$$

where the second inequality is due to the Markov inequality and Assumption 2(iii.a), and the third is due to Assumption 5(iii.c). As $z$ can be chosen arbitrarily small and the remaining terms are finite by assumption, we have $U_{T} \stackrel{p}{\rightarrow} 0$. The same argument is used to show $V_{T} \stackrel{p}{\rightarrow} 0$. Hence, $A_{1 T}=o_{p}(1)$ is proved.

Next, we show $A_{2 T}=o_{p}(1)$. For this, it suffices to show $A_{2 T, i, j}:=$ $\frac{1}{2 c_{T} T} \sum_{t=1}^{T}$

$1_{\left[-c_{T} \leq \varepsilon_{i, j, t} \leq c_{T}\right]}\left|\nabla_{h} \hat{q}_{i, j, t}-\nabla_{h} q_{i, j, t}^{*}\right| \times\left|\nabla_{s} \hat{q}_{i, j, t}\right|=o_{p}(1)$ for each $i$ and $j$. Note that

$$
\begin{aligned}
A_{2 T, i, j} & \leq \frac{1}{2 c_{T} T} \sum_{t=1}^{T}\left|\nabla_{h} \hat{q}_{i, j, t}-\nabla_{h} q_{i, j, t}^{*}\right| \times\left|\nabla_{s} \hat{q}_{i, j, t}\right| \\
& \leq \frac{1}{2 c_{T} T} \sum_{t=1}^{T}\left\|\nabla_{h}^{2} q_{i, j, t}(\cdot, \tilde{\alpha})\right\| \times \| \hat{\alpha}_{T}-\alpha^{*}|| \times\left|\nabla_{s} \hat{q}_{i, j, t}\right| \\
& \leq \frac{1}{2 c_{T}}\left\|\hat{\alpha}_{T}-\alpha^{*}\right\| \frac{1}{T} \sum_{t=1}^{T} D_{2, t} D_{1, t} \\
& =\frac{1}{2 c_{T} T^{1 / 2}} T^{1 / 2}\left\|\hat{\alpha}_{T}-\alpha^{*}\right\| \frac{1}{T} \sum_{t=1}^{T} D_{2, t} D_{1, t},
\end{aligned}
$$


where $\tilde{\alpha}$ is between $\hat{\alpha}_{T}$ and $\alpha^{*}$, and $\nabla_{h}^{2} q_{j, t}(\cdot, \tilde{\alpha})$ is the first derivative of $\nabla_{h} \hat{q}_{j, t}$ with respect to $\alpha$, which is evaluated at $\tilde{\alpha}$. The last expression above is $o_{p}(1)$ because: (i) $T^{1 / 2}\left\|\hat{\alpha}_{T}-\alpha^{*}\right\|=O_{p}(1)$ by Theorem 2 ; (ii) $T^{-1} \sum_{t=1}^{T} D_{2, t} D_{1, t}=$ $O_{p}(1)$ by the ergodic theorem; and (iii) $1 /\left(c_{T} T^{1 / 2}\right)=o_{p}(1)$ by Assumption 7 (iii). Hence, $A_{2 T}=o_{p}(1)$. The other claims $A_{3 T}=o_{p}(1)$ and $A_{4 T}=$ $O_{p}(1)$ can be analogously and more easily proven. Hence, they are omitted. Therefore, we finally have $Q_{T}-\hat{Q}_{T} \stackrel{p}{\rightarrow} 0$, which, together with (21) and (22), implies that $\hat{Q}_{T}-Q^{*} \stackrel{p}{\rightarrow} 0$. As a result, the proof is complete. 
Table 1 - Financial institutions included in the sample NAME

77 BANK

COUNTRY SECTOR

2 ALLIED IRISH BANKS

3 ALPHA BANK

AUS.AND NZ.BANKING GP.

5 AWA BANK

BANK OF IRELAND

BANKINTER 'R'

BARCLAYS

BB\&T

0 BANCA CARIGE

1 BANCA MONTE DEI PASCHI

2 BANCA POPOLARE DI MILANO

BANCA PPO.DI SONDRIO

4 BANCA PPO.EMILIA ROM.

15 BBV.ARGENTARIA

16 BANCO COMR.PORTUGUES 'R'

BANCO DE VALENCIA

18 BANCO ESPIRITO SANTO

19 BANCO POPOLARE

20 BANCO POPULAR ESPANOL

21 BANCO SANTANDER

22 BNP PARIBAS

23 BANK OF AMERICA

24 BANK OF EAST ASIA

25 BANK OF KYOTO

26 BANK OF MONTREAL

27 BK.OF NOVA SCOTIA

8 BANK OF QLND.

BANK OF YOKOHAMA

BENDIGO \& ADELAIDE BANK

COMMERZBANK (XET)

CREDIT SUISSE GROUP N

CREDITO VALTELLINES

4 CANADIAN IMP.BK.COM.

CHIBA BANK

CHUGOKU BANK

CHUO MITSUI TST.HDG.

CITIGROUP

COMERICA

1 DANSKE BANK

2 DBS GROUP HOLDINGS

43 DEUTSCHE BANK (XET)

44 DEXIA

45 DNB NOR

46 DAISHI BANK

47 EFG EUROBANK ERGASIAS

48 ERSTE GROUP BANK

49 FIFTH THIRD BANCORP

\begin{tabular}{ll} 
JP & BK \\
IE & BK \\
GR & BK \\
AU & BK \\
JP & BK \\
IE & BK \\
ES & BK \\
GB & BK \\
US & BK \\
IT & BK \\
IT & BK \\
IT & BK \\
IT & BK \\
IT & BK \\
ES & BK \\
PT & BK \\
ES & BK \\
PT & BK \\
IT & BK \\
ES & BK \\
ES & BK \\
FR & BK \\
US & BK \\
HK & BK \\
JP & BK \\
CA & BK \\
CA & BK \\
AU & BK \\
JP & BK \\
AU & BK \\
DE & BK \\
CH & BK \\
IT & BK \\
CA & BK \\
JP & BK \\
JP & BK \\
JP & BK \\
US & BK \\
US & BK \\
AU & BK \\
DK & BK \\
SG & BK \\
DE & BK \\
BE & BK \\
NO & BK \\
JP & BK \\
GR & BK \\
AT & BK \\
US & BK \\
\hline &
\end{tabular}

NAME

KA FINANCIAL GP.

51 SOCIETE GENERALE

52 GUNMA BANK

53 HSBC HOLDINGS

54 HACHIJUNI BANK

55 HANG SENG BANK

56 HIGO BANK

57 HIROSHIMA BANK

58 HOKUHOKU FINL. GP.

59 HUDSON CITY BANC

60 HUNTINGTON BCSH.

61 HYAKUGO BANK

HYAKUJUSHI BANK

3 INTESA SANPAOLO

64 IYO BANK

65 JP MORGAN CHASE \& CO.

66 JYSKE BANK

67 JOYO BANK

68 JUROKU BANK

69 KBC GROUP

70 KAGOSHIMA BANK

71 KEIYO BANK

72 KEYCORP

73 LLOYDS BANKING GROUP

74 M\&T BK.

MEDIOBANCA

MARSHALL \& ILSLEY

77 MIZUHO TST \& BKG.

78 NATIONAL BK.OF GREECE

9 NATIXIS

80 NORDEA BANK

81 NANTO BANK

82 NATIONAL AUS.BANK

83 NAT.BK.OF CANADA

84 NY.CMTY.BANC

85 NISHI-NIPPON CITY BANK

86 NORTHERN TRUST

87 OGAKI KYORITSU BANK

88 OVERSEA-CHINESE BKG.

89 BANK OF PIRAEUS

90 PNC FINL SVS.GP.

91 POHJOLA PANKKI A

92 PEOPLES UNITED FINANCIAL

93 ROYAL BANK OF SCTL.GP.

94 REGIONS FINL.NEW

95 RESONA HOLDINGS

96 ROYAL BANK CANADA

97 SEB 'A'

98 STANDARD CHARTERED

\begin{tabular}{|c|c|c|c|}
\hline COUNTRY & SECTOR & & NAME \\
\hline JP & BK & 99 & SVENSKA HANDBKN.'A' \\
\hline FR & BK & 100 & SWEDBANK 'A' \\
\hline JP & BK & 101 & SYDBANK \\
\hline HK & BK & 102 & SAN-IN GODO BANK \\
\hline JP & BK & 103 & SHIGA BANK \\
\hline HK & BK & 104 & SHINKIN CENTRAL BANK PF. \\
\hline JP & BK & 105 & SUMITOMO MITSUI FINL.GP. \\
\hline JP & BK & 106 & SUMITOMO TRUST \& BANK. \\
\hline JP & BK & 107 & SUNTRUST BANKS \\
\hline US & BK & 108 & SUNCORP-METWAY \\
\hline US & BK & 109 & SURUGA BANK \\
\hline JP & BK & 110 & TORONTO-DOMINION BANK \\
\hline JP & BK & 111 & US BANCORP \\
\hline IT & BK & 112 & UBS 'R' \\
\hline $\mathrm{JP}$ & BK & 113 & UNICREDIT \\
\hline US & BK & 114 & UNITED OVERSEAS BANK \\
\hline DK & BK & 115 & VALIANT 'R' \\
\hline JP & BK & 116 & WELLS FARGO \& CO \\
\hline JP & BK & 117 & WESTPAC BANKING \\
\hline $\mathrm{BE}$ & BK & 118 & WING HANG BANK \\
\hline JP & BK & 119 & YAMAGUCHI FINL.GP. \\
\hline JP & BK & 120 & 3I GROUP \\
\hline US & BK & 121 & ABERDEEN ASSET MAN. \\
\hline GB & BK & 122 & ACKERMANS \& VAN HAAREN \\
\hline US & BK & 123 & AMP \\
\hline IT & BK & 124 & ASX \\
\hline US & BK & 125 & ACOM \\
\hline $\mathrm{JP}$ & BK & 126 & AMERICAN EXPRESS \\
\hline GR & BK & 127 & BANK OF NEW YORK MELLON \\
\hline FR & BK & 128 & BLACKROCK \\
\hline SE & BK & 129 & CI FINANCIAL \\
\hline $\mathrm{JP}$ & BK & 130 & CLOSE BROTHERS GROUP \\
\hline AU & BK & 131 & CIE.NALE.A PTF. \\
\hline CA & BK & 132 & CRITERIA CAIXACORP \\
\hline US & BK & 133 & CHALLENGER FINL.SVS.GP. \\
\hline JP & BK & 134 & CHARLES SCHWAB \\
\hline US & BK & 135 & CHINA EVERBRIGHT \\
\hline JP & BK & 136 & COMPUTERSHARE \\
\hline SG & BK & 137 & CREDIT SAISON \\
\hline GR & BK & 138 & DAIWA SECURITIES GROUP \\
\hline US & BK & 139 & EURAZEO \\
\hline FI & BK & 140 & EATON VANCE NV. \\
\hline US & BK & 141 & EQUIFAX \\
\hline GB & BK & 142 & FRANKLIN RESOURCES \\
\hline US & BK & 143 & GAM HOLDING \\
\hline JP & BK & 144 & GBL NEW \\
\hline CA & BK & 145 & GOLDMAN SACHS GP. \\
\hline $\mathrm{SE}$ & BK & 146 & ICAP \\
\hline GB & BK & 147 & IGM FINL. \\
\hline
\end{tabular}

COUNTRY SECTOR

SE

SE BK

BK

$\mathrm{JP} \quad \mathrm{BK}$

JP BK

JP $\quad$ BK

US $\quad$ BK

AU BK

CA BK

US BK

$\mathrm{CH} \quad \mathrm{BK}$

SG $\quad$ BK

$\mathrm{CH} \quad \mathrm{BK}$

US $\quad$ BK

$\mathrm{HK} \quad \mathrm{BK}$

JP BK

$\begin{array}{ll}\text { GB } & \text { FS } \\ \text { GB } & \text { FS }\end{array}$

BE $\quad$ FS

AU FS

AU FS

US FS

$\begin{array}{ll}\text { US } & \text { FS } \\ \text { US } & \text { FS }\end{array}$

CA FS

BE $\quad$ FS

$\begin{array}{ll}\text { ES } & \text { FS }\end{array}$

AU FS

$\begin{array}{ll}\text { US } & \text { FS } \\ \text { HK } & \text { FS }\end{array}$

AU FS

JP FS

$\begin{array}{ll}\text { JP } & \text { FS } \\ \text { FR } & \text { FS }\end{array}$

US FS

$\begin{array}{ll}\text { US } & \text { FS }\end{array}$

$\mathrm{CH} \quad \mathrm{FS}$

BE $\quad F S$

$\begin{array}{ll}\text { US } & \text { FS } \\ \text { GB } & \text { FS }\end{array}$
$B K$
$B K$
$B K$
$B K$
$B K$
$B K$
$B K$
$B K$
$B K$
$B K$
$B K$
$B K$
$B K$
$B K$
$B K$
$B K$
$B K$
$B K$
$B K$
$B K$
$F S$
$F S$
$F S$
$F S$
$F S$
$F S$
$F S$
$F S$
$F S$
$F S$
$F S$
$F S$
$F S$
$F S$
$F S$
$F S$
$F S$
$F S$
$F S$
$F S$
$F S$
$F S$
$F S$
$F S$
$F S$
$F S$
$F S$ 
NAME

148 INDUSTRIVARDEN 'A'

149 INTERMEDIATE CAPITAL GP.

150 KINNEVIK 'B'

151 INVESTOR 'B'

152 LEGG MASON

153 MAN GROUP

154 MARFIN INV.GP.HDG

55 MACQUARIE GROUP

156 MITSUB.UFJ LSE.\& FINANCE

158 MOODY'S

159 MORGAN STANLEY

160 NOMURA HDG

161 ORIX

162 PARGESA 'B'

163 PROVIDENT FINANCIAL

164 PERPETUAL

165 RATOS 'B'

166 SCHRODERS

167 SLM

168 SOFINA

169 STATE STREET

170 T ROWE PRICE GP.

171 TD AMERITRADE HOLDING

172 WENDEL

173 ACE

174 AEGON

175 AFLAC
157 MIZUHO SECURITIES
NAME

176 AGEAS (EX-FORTIS)

177 ALLIANZ (XET)

179 AON

180 GENERALI

181 AVIVA

182 AXA ASIA PACIFIC HDG.

183 AXA

184 ALLSTATE

186 ARCH CAP.GP.

187 BALOISE-HOLDING AG

188 BERKSHIRE HATHAWAY 'B'

189 CNP ASSURANCES

190 CHUBB

191 CINCINNATI FINL.

193 FAIRFAX FINL.HDG.

194 GREAT WEST LIFECO

195 HANNOVER RUCK. (XET)

197 HARTFORD FINL.SVS.GP.

199 JARDINE LLOYD THOMPSON

200 LEGAL \& GENERAL

201 LINCOLN NAT.

202 LOEWS

203 MAPFRE
196 HELVETIA HOLDING N

NAME

204 MS\&AD INSURANCE GP.HDG.

COUNTRY

207 MARKEL

208 MARSH \& MCLENNAN

209 OLD MUTUAL

210 PRUDENTIAL

211 PARTNERRE

212 POWER CORP.CANADA

213 POWER FINL.

214 PROGRESSIVE OHIO

215 QBE INSURANCE GROUP

216 RSA INSURANCE GROUP

217 RENAISSANCERE HDG.

218 SAMPO 'A'

219 SCOR SE

221 SWISS LIFE HOLDING

222 SWISS RE 'R'

223 TOPDANMARK

224 TORCHMARK

225 TRAVELERS COS.

226 UNUM GROUP

227 VIENNA INSURANCE GROUP A

228 W R BERKLEY

229 XL GROUP

230 ZURICH FINANCIAL SVS.

$\begin{array}{ll}\text { JP } & \text { IN } \\ \text { DE } & \text { IN } \\ \text { CA } & \text { IN } \\ \text { US } & \text { IN } \\ \text { US } & \text { IN } \\ \text { GB } & \text { IN } \\ \text { GB } & \text { IN } \\ \text { US } & \text { IN } \\ \text { CA } & \text { IN } \\ \text { CA } & \text { IN } \\ \text { US } & \text { IN } \\ \text { AU } & \text { IN } \\ \text { GB } & \text { IN } \\ \text { US } & \text { IN } \\ \text { FI } & \text { IN } \\ \text { FR } & \text { IN } \\ \text { NO } & \text { IN } \\ \text { CH } & \text { IN } \\ \text { CH } & \text { IN } \\ \text { DK } & \text { IN } \\ \text { US } & \text { IN } \\ \text { US } & \text { IN } \\ \text { US } & \text { IN } \\ \text { AT } & \text { IN } \\ \text { US } & \text { IN } \\ \text { US } & \text { IN } \\ \text { CH } & \text { IN } \\ & \end{array}$

Note: The abbreviation for the sector classification are as follows: BK = Bank, FS = Financial Services, IN = Insurance. Classification as provided by Datastream. 
Table 2 - Breakdown of financial institutions by sector and by geographic area

\begin{tabular}{l|ccc|c}
\hline & Banks & Financial Services & Insurances & \\
\hline EU & 47 & 22 & 27 & $\mathbf{9 6}$ \\
North America & 25 & 17 & 28 & $\mathbf{7 0}$ \\
Asia & 47 & 14 & 3 & $\mathbf{6 4}$ \\
\hline & $\mathbf{1 1 9}$ & $\mathbf{5 3}$ & $\mathbf{5 8}$ & $\mathbf{2 3 0}$ \\
\hline
\end{tabular}

Note: Swiss and Norwegian financial institutions have been classified as EU. Asia includes Australian financial institutions.

Table 3 - Estimates and standard errors for selected financial institutions

\section{Barclays}

\begin{tabular}{lccccc}
\hline & $c_{1}$ & $a_{11}$ & $a_{12}$ & $b_{11}$ & $b_{12}$ \\
\hline & $\mathbf{- 0 . 1 9}$ & $\mathbf{- 0 . 4 5}$ & -0.12 & $\mathbf{0 . 7 7}$ & -0.01 \\
s.e. & $\mathbf{0 . 0 7}$ & $\mathbf{0 . 1 4}$ & 0.09 & $\mathbf{0 . 0 6}$ & 0.01 \\
\hline & $C_{2}$ & $a_{21}$ & $a_{22}$ & $b_{21}$ & $b_{22}$ \\
\hline & $\mathbf{- 0 . 1 7}$ & $\mathbf{- 0 . 4 0}$ & -0.22 & $\mathbf{- 0 . 2 1}$ & $\mathbf{0 . 9 6}$ \\
s.e. & $\mathbf{0 . 0 9}$ & $\mathbf{0 . 1 3}$ & 0.12 & $\mathbf{0 . 0 8}$ & $\mathbf{0 . 0 1}$ \\
\hline
\end{tabular}

\section{Deutsche Bank}

\begin{tabular}{cccccc}
\hline & $c_{1}$ & $a_{11}$ & $a_{12}$ & $b_{11}$ & $b_{12}$ \\
\hline & $\mathbf{0 . 1 2}$ & $\mathbf{- 0 . 3 7}$ & -0.07 & $\mathbf{0 . 8 7}$ & -0.03 \\
s.e. & $\mathbf{0 . 0 5}$ & $\mathbf{0 . 1 5}$ & 0.04 & $\mathbf{0 . 0 5}$ & 0.02 \\
\hline & $c_{2}$ & $a_{21}$ & $a_{22}$ & $b_{21}$ & $b_{22}$ \\
\hline & -0.16 & -0.11 & -0.32 & -0.03 & $\mathbf{0 . 8 7}$ \\
s.e. & 0.11 & 0.34 & 0.22 & 0.11 & $\mathbf{0 . 0 8}$ \\
\hline
\end{tabular}

Citigroup

\begin{tabular}{cccccc}
\hline & $c_{1}$ & $a_{11}$ & $a_{12}$ & $b_{11}$ & $b_{12}$ \\
\hline & -0.13 & -0.08 & $\mathbf{- 0 . 1 5}$ & $\mathbf{0 . 8 3}$ & 0.02 \\
s.e. & 0.08 & 0.08 & $\mathbf{0 . 0 5}$ & $\mathbf{0 . 0 9}$ & 0.02 \\
\hline & $c_{2}$ & $a_{21}$ & $a_{22}$ & $b_{21}$ & $b_{22}$ \\
\hline & -0.02 & -0.10 & -0.17 & -0.06 & $\mathbf{0 . 9 7}$ \\
s.e. & 0.10 & 0.13 & 0.11 & 0.12 & $\mathbf{0 . 0 3}$ \\
\hline
\end{tabular}

Goldman Sachs

\begin{tabular}{cccccc}
\hline & $c_{1}$ & $a_{11}$ & $a_{12}$ & $b_{11}$ & $b_{12}$ \\
\hline & -0.03 & $\mathbf{- 0 . 1 3}$ & $\mathbf{- 0 . 1 1}$ & $\mathbf{0 . 9 5}$ & -0.04 \\
s.e. & 0.02 & $\mathbf{0 . 0 5}$ & $\mathbf{0 . 0 5}$ & $\mathbf{0 . 0 2}$ & 0.02 \\
\hline & $c_{2}$ & $a_{21}$ & $a_{22}$ & $b_{21}$ & $b_{22}$ \\
\hline & -0.04 & -0.03 & $\mathbf{- 0 . 1 7}$ & 0.00 & $\mathbf{0 . 9 3}$ \\
s.e. & 0.03 & 0.11 & $\mathbf{0 . 0 7}$ & 0.04 & $\mathbf{0 . 0 3}$ \\
\hline
\end{tabular}

Note: Coefficients significant at the $5 \%$ level formatted in bold. 
Figure 1 - 1\% quantile for selected financial institutions

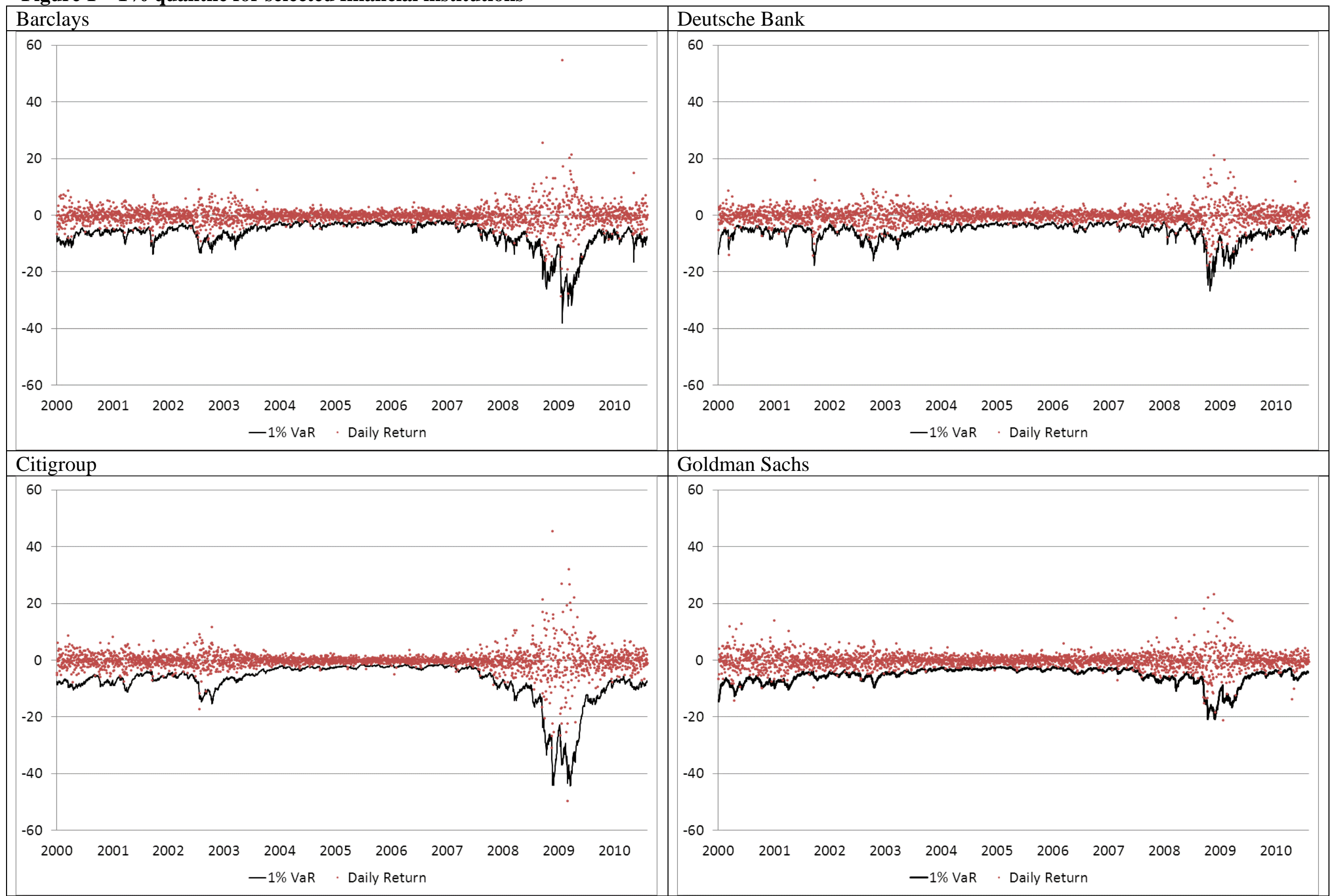


Figure 2 - Impulse-response functions to a shock to the market for selected financial institutions

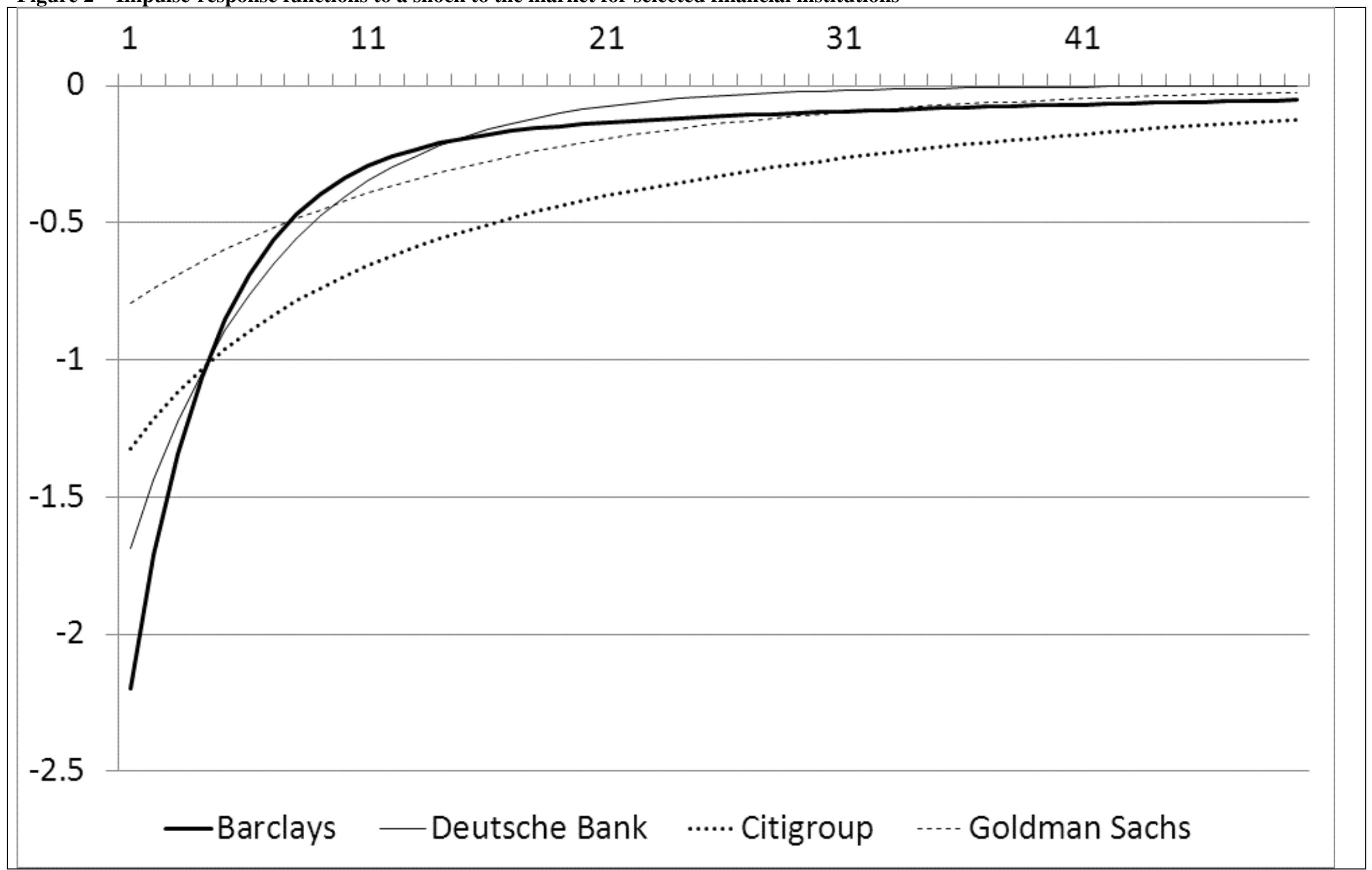


Figure 3 - Impulse-response functions by sectoral and geographic aggregation

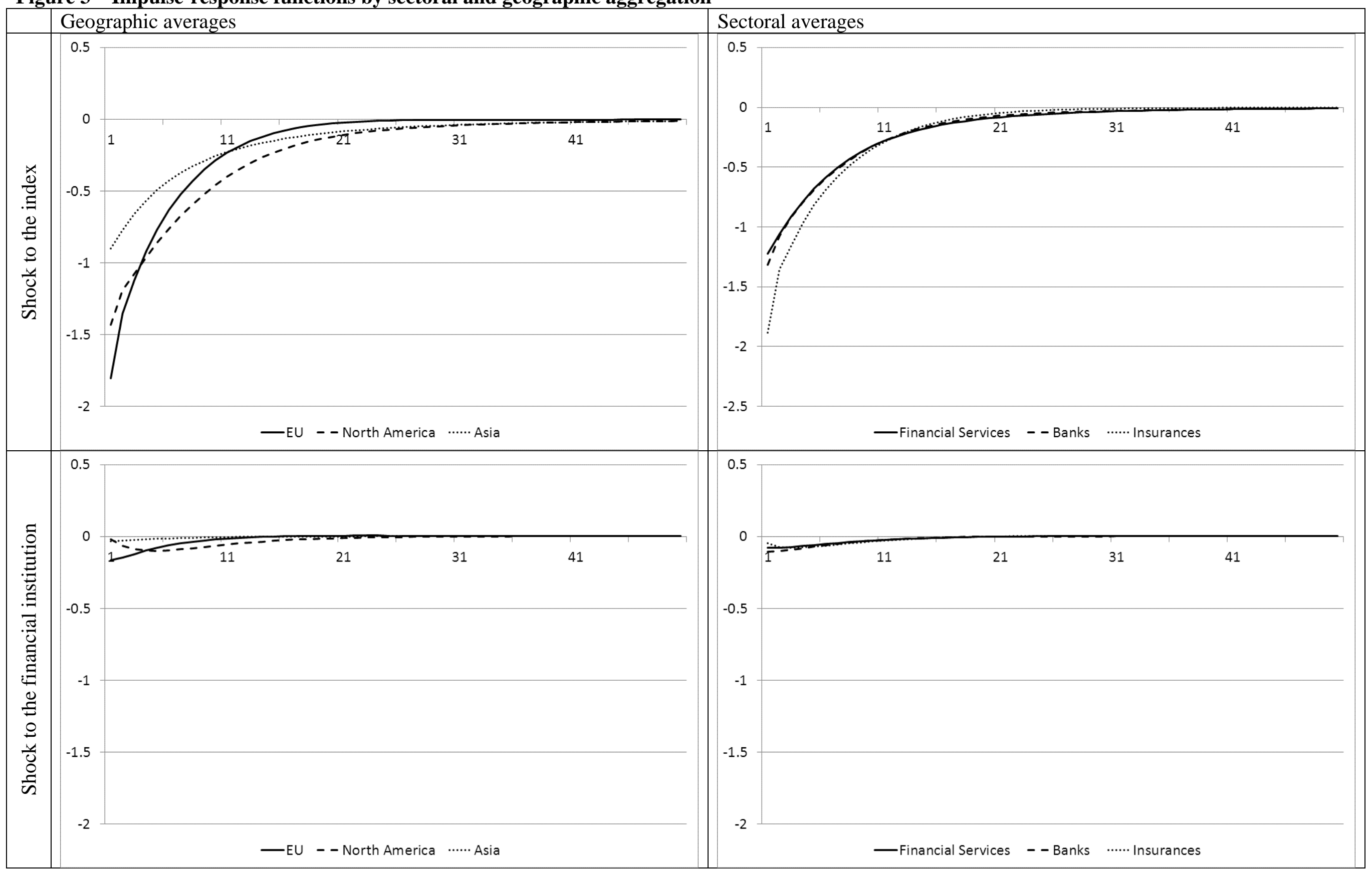




\section{Figure 4 - Strongest and weakest VaR impulse-responses}

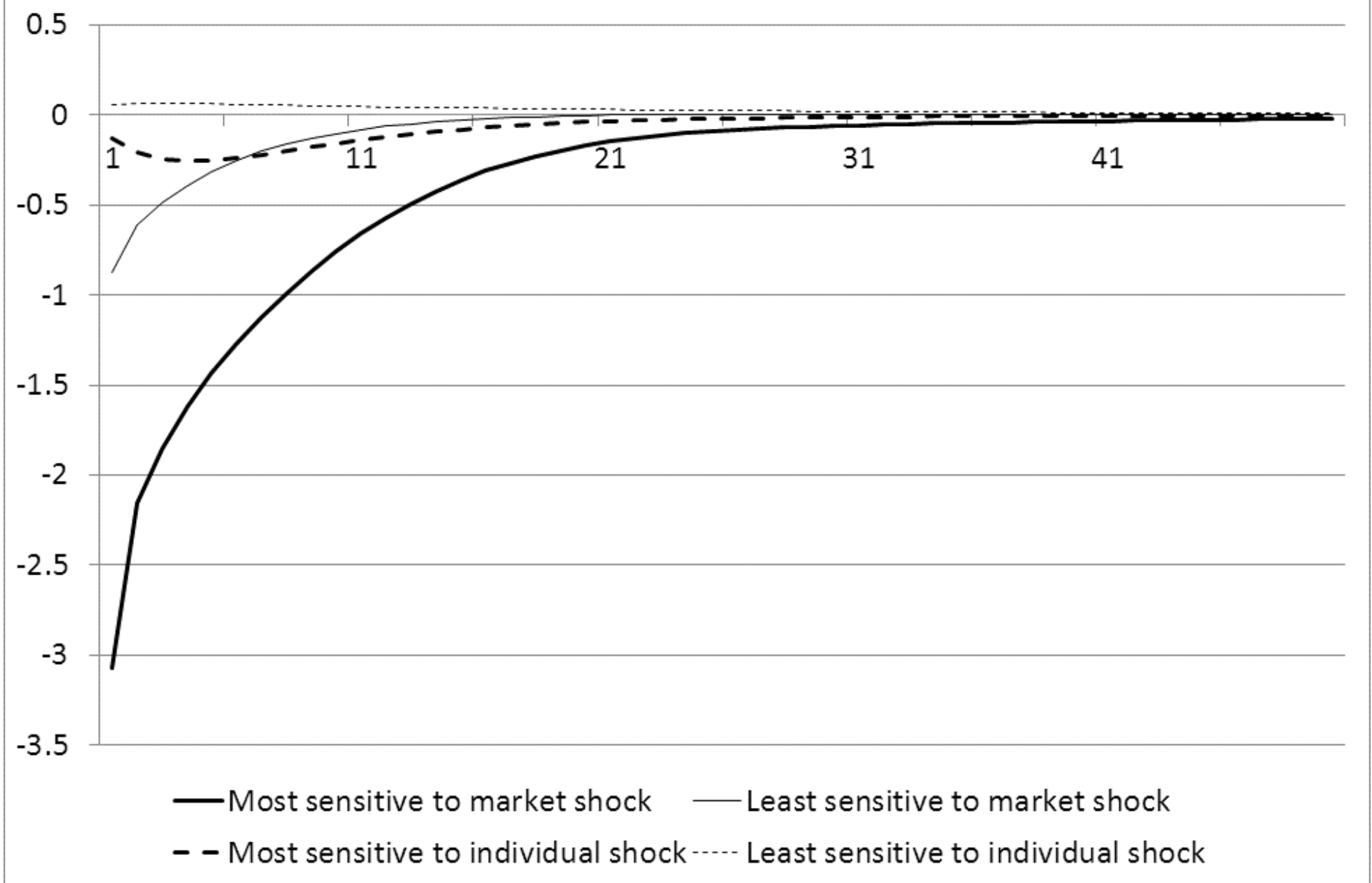

Note: The figure reports the average impulse-response function of the 20 financial institutions with the strongest and weakest impact, as measured by the area below the impulse-response function. 
Figure 5 - In-sample and out-of-sample average VaR and price developments of the 20 financial institutions with the strongest and weakest VaR impulse-responses to a market shock

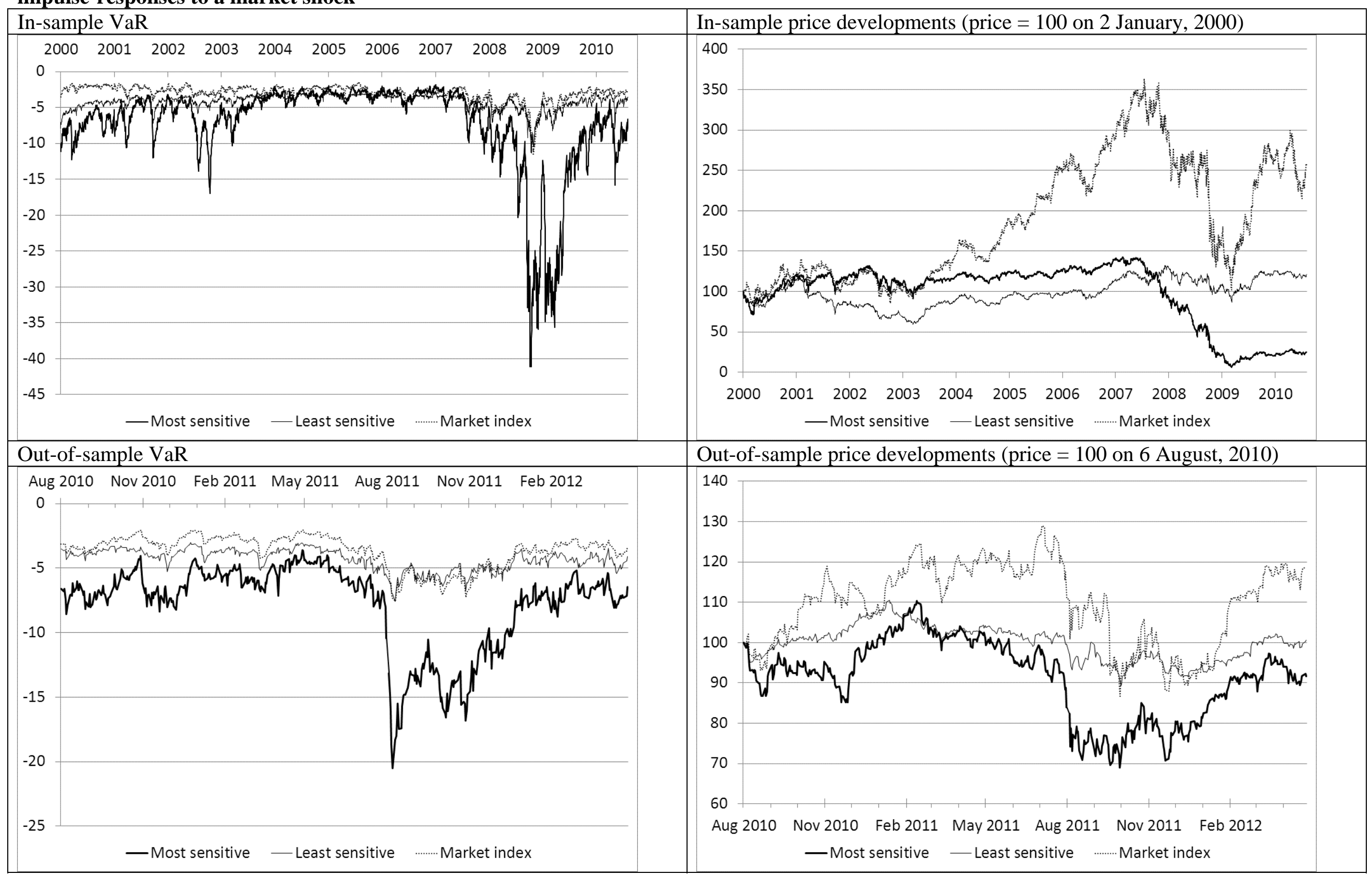

